\title{
The growing threat of light pollution to ground-based observatories
}

\author{
Richard F. Green ${ }^{1}$ (D) Christian B. Luginbuhl ${ }^{2} \cdot$ Richard J. Wainscoat $^{3}$. \\ Dan Duriscoe 4
}

Received: 15 May 2021 / Accepted: 16 November 2021 / Published online: 27 January 2022

(C) The Author(s) 2022

\begin{abstract}
Human activity is rapidly increasing the negative impact of artificial skyglow at even the most remote professional observatory sites. Assessment of the actual impact requires an understanding of the propagation as a function of source spectral energy distribution. The higher blue content of light-emitting diodes being widely used as replacement for sodium discharge lamps has greater impact closer to the source, and less impact for more distant mountain-top sites. All-sky cameras with moderate angular resolution provide data and metrics sufficient to model and remove celestial contributions and provide measures of artificial light contribution. The natural skyglow is significantly affected by solar activity, which must be accounted for in determining secular trends in the artificial component. With the availability of the New World Atlas of the Artificial Sky Brightness, a direct comparison is made of the modeled artificial contribution to the sites with the largest aperture telescopes, noting the possible systematic errors in individual cases. Population growth of the nearest urban centers allows a prediction of the change in that brightness over a decade. All site protections are effected primarily by national or regional regulation. A collection of worldwide regulations shows that most are leveraged off environmental protection statutes, while in the U.S., they are largely based on land-use zones. Particular examples are presented in more detail for Flagstaff, Arizona, and the Island of Hawai'i. The latest rapidly growing threat is that of reflected sunlight from large constellations of satellites in low-earth orbit. A snapshot is provided of that rapidly changing situation. In all cases, astronomers must become very proactive in educating the public about the cultural value of visual or naked eye astronomy as well as the science and the need for access to a dark night sky for astronomical research.
\end{abstract}

Keywords Light pollution · Methods - Observational · Atmospheric effects 


\section{Contents}

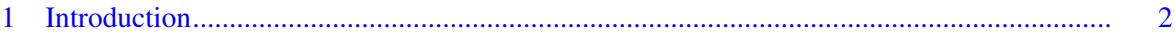

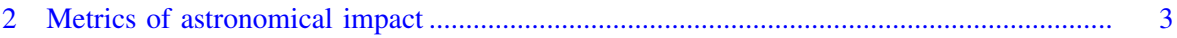

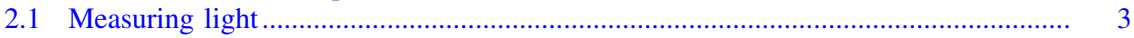

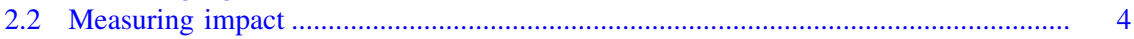

3 Propagation of artificial light and dependence on source type ................................ 5

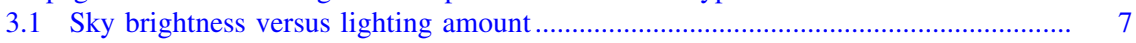

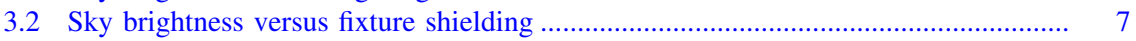

3.3 Sky brightness versus distance ..................................................................... 9

3.4 Sky brightness versus lamp spectrum .................................................................... 9

4 Field measurements of artificial night sky brightness ................................................ 13

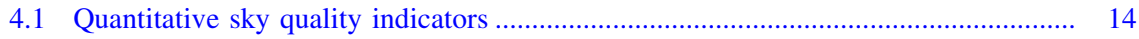

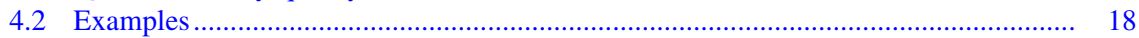

5 Sky brightness measurements and impact of artificial sources .................................... 22

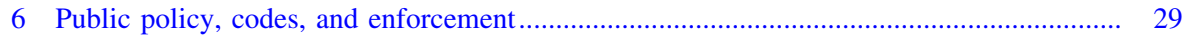

6.1 Light pollution/lighting regulation ............................................................. 29

6.2 Two detailed examples ........................................................................... 35

6.2.1 Flagstaff, Arizona USA......................................................................... 35

6.2.2 Maunakea, Hawaii USA …................................................................... 36

6.2.2.1 Approaches to local regulation ............................................... 36

6.2.2.2 Results in night sky protection ........................................... 39

7 Satellite constellations in low-Earth orbit ............................................................... 41

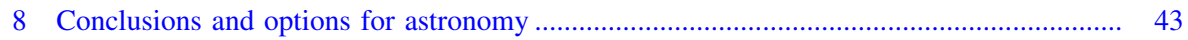

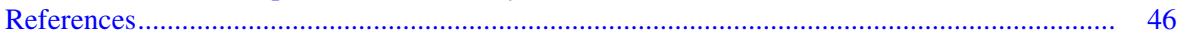

\section{Introduction}

The growth of artificial light at night (ALAN) worldwide has drastically diminished the accessibility of dark skies to all members of modern cultures, and for professional astronomy, and threatens even remote professional observatory sites with increased anthropogenic skyglow. In the past decade, the globally averaged increase was $2 \%$ per year in both lit area and total radiance (Kyba et al. 2017). That rate is roughly double the rate of world population growth. Factors contributing to the more rapid rate of ALAN increase include economic growth faster than population growth and the reduction of illumination cost, sometimes leading to elastic demand (Kyba et al. 2017).

A general definition of light pollution is "any adverse effect or impact attributable to the use of artificial light at night". Artificial skyglow is the form of light pollution impacting ground-based observatories. Many of the professional observatories with 4-m class telescopes were built decades ago in proximity to towns with modest populations. In the subsequent time, many of those regions have experienced substantial population growth with accompanying increase in ALAN. Human activity generating ALAN in remote regions near the newest professional observatories include open-pit mining, military exercises, highway infrastructure, border control, and over-flights.

This paper provides an overview of the issues, requirements and approaches for measurements of impact, focusing on impacts on astronomy and the current 


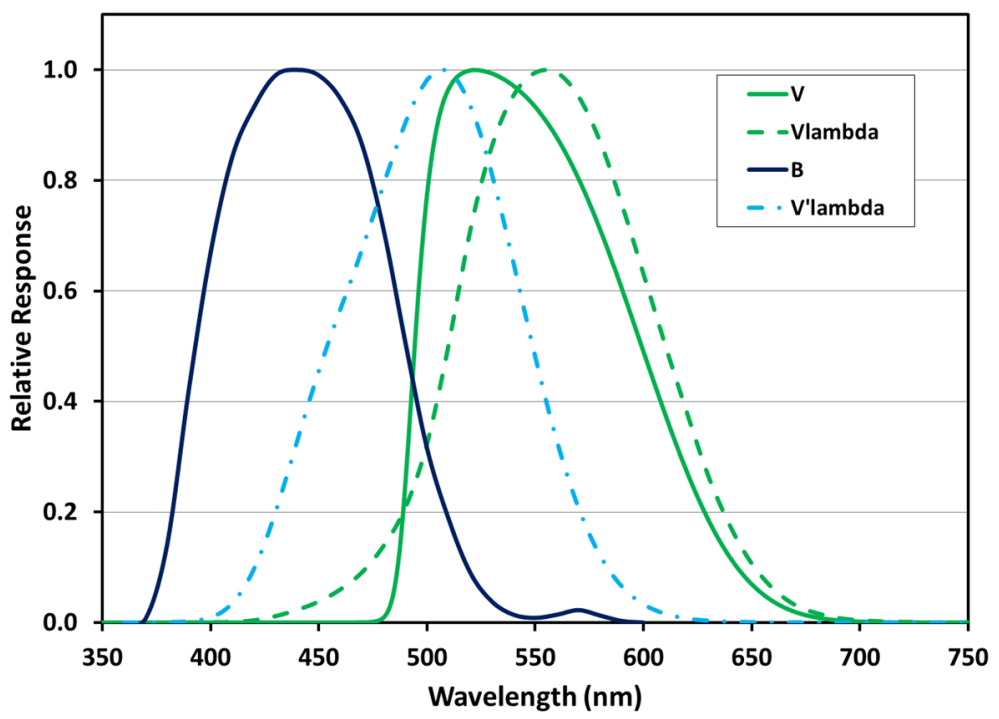

Fig. 1 Spectral response of the Johnson $\mathrm{V}$ and $\mathrm{B}$ passbands, as well as the human light-adapted (photopic_-V lambda) and dark-adapted (scotopic $\mathrm{V}$ ' lambda) responses

regulatory frameworks applied locally for protection of major observatories, and a brief description of the new problem of satellite constellations.

\section{Metrics of astronomical impact}

\subsection{Measuring light}

Electromagnetic radiation, including light pollution, is characterized by radiant flux as a function of wavelength, or spectral intensity. In the context of lighting and light pollution, the wavelengths of interest cover the visible spectrum, or approximately 450 through $670 \mathrm{~nm}$. The full range of ground-based astronomical coverage extends to the atmospheric cutoff at $\sim 300 \mathrm{~nm}$. Redward of $700 \mathrm{~nm}$, skyglow from $\mathrm{OH}$ increasingly dominates that of any artificial contribution. Flux can be quantified either as a function of wavelength or integrated within particular portions of the spectrum. For lighting design purposes, outdoor lighting is measured in terms of the spectral response of the (light adapted) human eye (cf. Fig. 1), with luminous flux measured in "lumens," illumination levels in lux (lumens per square meter), and brightness or "luminance" in candela (lumens per steradian) or candela per square meter. ${ }^{1}$

In astronomical discourse, the quantity of artificial light affecting astronomical measurement has most commonly been measured using the Johnson V bandpass, which also closely approximates the human photopic response used to define luminous flux (Fig. 1). This spectral region has been used historically by

\footnotetext{
1 also called "nits".
} 
astronomers as a matter of convenience, not only because it covers a significant portion of the visual spectrum and thus the portion of the spectrum principally affected by artificial lighting, but also because of the historical importance of the Johnson photometric system. Thus light pollution and generally sky brightness measures have most commonly been expressed in V astronomical magnitudes per square arc second, though Garstang's (1986) treatments also used "nano lamberts," [footnote: 1 lambert $(\mathrm{L})=1 / \pi$ candela per square centimeter] which has often continued in modeling discussions. ${ }^{2}$ Some discussion has also been developed around measurements in the Johnson B band (e.g. Garstang 1989b), or other passbands relevant to various astronomical photometric systems, which have exclusively used astronomical magnitudes per square arc second. Bará et al. (2020a, b) discuss the impact of color terms from various night sky conditions on the photometric transformations.

Besides measurements of lighting amount, the other pertinent metric for measuring light is wavelength, which can be understood both as the particular wavelengths of emission as well as the sum of all wavelengths-or "bandwidth"emitted by a source.

\subsection{Measuring impact}

When gauging impacts on astronomical measurements, different approaches have been taken. Discussions concerning the impact of an LPS to LED conversion in Hawaii have developed an approach where impact of proposed LED alternatives is measured through the proportion of the spectral emissions in the "blue" portion of the spectrum, defined as wavelength shortward of $500 \mathrm{~nm}$, or alternatively within the passband defined by the Sloan g'-filter. This approach has now been used in other areas/jurisdictions, sometimes expressed as the "percent blue" or ratio of the radiant flux shortward of $500 \mathrm{~nm}$ (or sometimes another reference wavelength such as $550 \mathrm{~nm}$ ) compared to the radiant flux over all wavelengths (cf. IDA 2010; Canary Islands standards in Table 5). Another approach arising out of the focus on the blue portion of the spectrum uses the "correlated color temperature" or CCT. CCT is defined as the temperature, in Kelvin, of a black body radiator exhibiting the same color or "hue" to the light-adapted human eye. CCT is properly applied only to light spectra that approximate the spectrum produced by a blackbody. While it is generally true that lamps with lower CCT have decreased blue content or visual sky brightness impacts compared to lamps with greater CCT, CCT does not accurately correlate with either the "percent blue" impact or visual sky glow impacts (see below).

A more generalized approach, suggested by Luginbuhl et al. (2014) and used in discussions concerning Flagstaff LED retrofits from LPS, focuses on the spectral bandwidth to gauge impact on professional astronomical work, and uses a separate metric based on scotopic brightness to gauge impact on visual night sky brightness.

\footnotetext{
2 for the relation between astronomical and lighting or "luminous" metrics, see Garstang (1986) Eq. 19.
} 
Using spectral bandwidth rather than focusing on a particular portion of the spectrum is "spectrally neutral," thus implicitly treating all wavelength regions equally.

For visual effects of light pollution, measuring detrimental impacts to "cultural" astronomy (the ability to see stars in the night sky with the human eye as the detector), the "scotopic to photopic" or $S / P$ ratio is used. If outdoor lighting is specified in terms of the luminous flux (measured in lumens), then the $S / P$ ratio can be used to compare the relative visual impacts of lamp alternatives. This metric, based as it is on a biological action spectrum (human rod response), is also an approximate surrogate for many biological or ecological impacts, as increased sensitivity to the short wavelength portion of the visible spectrum is a common attribute of many biological systems (IDA 2010).

At this point, acceptable or target threshold values have rarely been established for any of these metrics (see Sect. 6). Thus, efforts toward light pollution mitigation have focused only on minimizing impacts, with little or no discussion of what an acceptable impact might be, and thus no way to determine whether light pollution exceeds thresholds or has been successfully mitigated. Approaches to date have concentrated on individual fixtures or at most, individual parcels of land, rather than the summed regional impact on observatories for which the effect of mitigation attempts is most relevant.

In general discussions, the portions of the sky that are most important to professional and cultural astronomy may be distinguished. Most astronomical observatories consider the sky from the zenith down to zenith angle of approximately $70^{\circ}$ (airmass $\sim 3$ ) to be the area of greatest concern. For visual observers, the entire sky is of interest, and in some circumstances the area near the horizon may be considered of greater import because the standing casual human observer faces the horizon.

\section{Propagation of artificial light and dependence on source type}

Though the physics describing the interaction of light with molecules and aerosols is well understood since the work of Rayleigh and Mie in the late 19th and early twentieth centuries, applying it to produce accurate predictions of the sky brightness arising from lighting use on the ground can become an enormously complex problem in practice. Generally, in real outdoor lighting installations such as in urban areas, critical information on the light sources is not known, or only approximately known. The atmosphere, particularly over large areas relevant to the propagation of light pollution, may often be non-uniform and not accurately described by simple exponential variations of density or particle numbers with altitude.

A physical model describing light pollution through the interaction of artificial light with the atmosphere was first developed by Garstang (1986, 1989a, 1991). Garstang's model was the first to treat a realistic atmosphere in a curved-Earth geometry with realistic treatment of scattering in the atmosphere by molecules and aerosols, with different scale heights, with a generalized formulation allowing 
evaluation of sky brightness at any direction in the sky from an observation point located at an arbitrary location relative to the light sources.

Garstang's treatment simplified the problem by assuming (1) the vertical structure of the atmosphere was the same everywhere and characterized by exponentially decreasing density of molecules and aerosols (with different scale heights); (2) second-order scattering was approximately treated, and higher order scattering was entirely neglected; (3) light sources on the ground were described by a total luminous output (in lumens or photons per second), with an assumed angular intensity distribution based on a Lambertian ground reflection from a uniform and everywhere horizontal ground surface supplemented by direct emissions from unshielded light fixtures with an assumed intensity versus zenith angle; 4) no interaction of light after emission from the fixture or reflection from the ground with objects in the near-ground environment.

Garstang's models have been elaborated by a number of workers (Cinzano et al. 2001; Aubé et al. 2005; Kocifaj et al. 2007 et seq.; Luginbuhl et al. 2009b, Aubé et al. 2018). Most of these developments have sought to accommodate more accurately real-world variations in atmospheric and lighting characteristics, to allow prediction of sky glow levels in specific locations under specific circumstances, or to allow evaluation of the impact of different lighting characteristics (such as shielding, spectrum, angular intensity distribution).

As currently implemented, these models can be used to predict the artificial sky glow over the entire sky given the spectral radiant input from a lighting installation or a developed area. Due to their critical dependence on a large variety of often unknown or incompletely known factors-variations in aerosol distributions and optical characteristics both as a function of altitude and latitude/longitude; shielding characteristics/angular intensity distribution (particularly upward) of the light source(s); spectral reflectances of illuminated surfaces; blocking characteristics of the near-ground environment in the vicinity of the light sources-these predictive models are generally under-constrained and thus not useful to produce accurate absolute predictions of sky brightness without resorting to simplifying assumptions such as done by Garstang.

Nonetheless, all of these models give approximately equivalent results when input parameters are equivalent, particularly under clear sky (i.e. cloudless and low aerosol) conditions in the "astronomically useful" portion of the sky, i.e. at zenith angles less than about $70^{\circ}$. The relative impacts of a lighting installation, given a known lumen amount but different shielding or spectral characteristics, for example, can be more reliably assessed (e.g. Duriscoe, Luginbuhl and Elvidge 2014). Thus the state of light pollution modeling is sufficient to investigate the influence of specific lighting practices or lighting regulations on the resultant sky glow (Luginbuhl et al. 2009b; Duriscoe et al. 2014; Barentine et al. 2018).

The following subsections discuss the influence of critical lighting characteristics on artificial sky glow, and implications for effective light pollution mitigation strategies. 


\subsection{Sky brightness versus lighting amount}

All other factors being equal (e.g. distance, shielding, spectral power distribution, blocking - see below), the impact of the light source on the artificial sky brightness at an Observatory site or elsewhere is directly proportional to the radiant or luminous flux of the light source. This is no more than a statement of conservation of energy in a light pollution context. Though this observation would seem almost too obvious to mention, its importance cannot be overstated in a cultural context where more lighting is often pressed for marketing or perceived safety reasons. Managing lighting amounts and locations presents enormous technical and political challenges (see Sect. 6), and apart from the establishment of lighting recommendations for particular applications through professional or national standards (e.g. roadway lighting), and a few important examples of overall limits on lighting amounts independent of land-use in communities with significant astronomical facilities, has not often been attempted.

\subsection{Sky brightness versus fixture shielding}

Light emanating directly upward from incompletely shielded fixtures has a disproportionate impact on sky brightness. This arises from two factors: (1) such light suffers no diminution due to (partial) reflection off of surfaces, and (2) light arising from such unshielded fixtures is typically directed at small angles above the horizontal [footnote: there is of course some lighting that is directed at much higher angles, sometimes nearly straight up, such as on billboards. Such lighting does not dominate direct uplight emission in the typical municipality]. Since the use of unshielded fixtures can in many circumstances also be viewed as unnecessary or wasteful, light pollution control efforts direct much attention at improving shielding.

Beginning with Garstang (1986), most light pollution researchers have found an average reflectivity of $15 \%$ is representative of illuminated surfaces (e.g. concrete, asphalt, vegetation, etc.). Thus, direct upward emissions, which suffer no such reflections, can be expected to have an impact $\sim 1 / 0.15=6.7 \times$ greater than an equal flux directed downward, neglecting for the moment the angular effects. Since direct upward emission fractions average about 10\% (Garstang 1986, 1991; Luginbuhl et al 2009a), light flux propagating upward is very roughly equally split between direct and reflected emissions (i.e., 90\% downward $\times 15 \%$ reflectivity $=13.5 \%$ ). But due to the increased impact of light rays directed just above the horizontal, the impact of the direct upward emissions on e.g. sky brightness is in most cases dramatically greater than reflected upward emissions.

Compared to an equal flux directed downward, Luginbuhl et al. (2009a) predicted that direct upward emissions can cause 40 to over 150 times the zenith sky glow impact of an equal flux directed downward (Fig. 2) when observed at some distance from the light sources, and in environments where there is little blocking. In realistic environments with near-ground blocking by structures and vegetation, these ratios will be decreased significantly, but remain much greater than one. These predictions are supported by measures reported by Falchi (2011), who found the influence of direct upward emission to be $\sim 1.5$ to $\sim 16$ times greater than reflected upward 


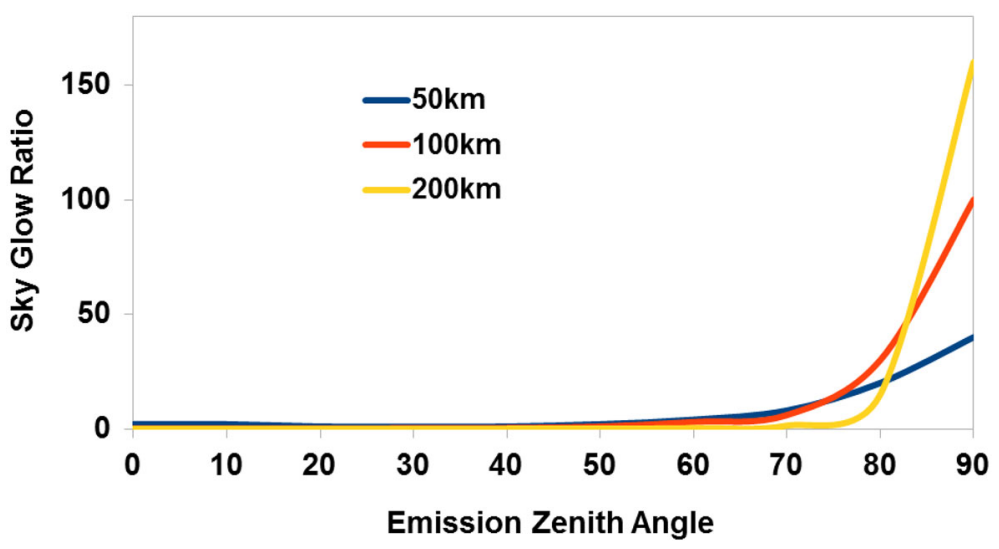

Fig. 2 Overhead sky brightness impact of luminous flux directed into the indicated zenith angles, compared to an equal luminous flux directed downward onto a Lambertian surface of $15 \%$ reflectivity, as observed from 50, 100 and $200 \mathrm{~km}$ from the light source (from Luginbuhl et al. 2009a, b, c)

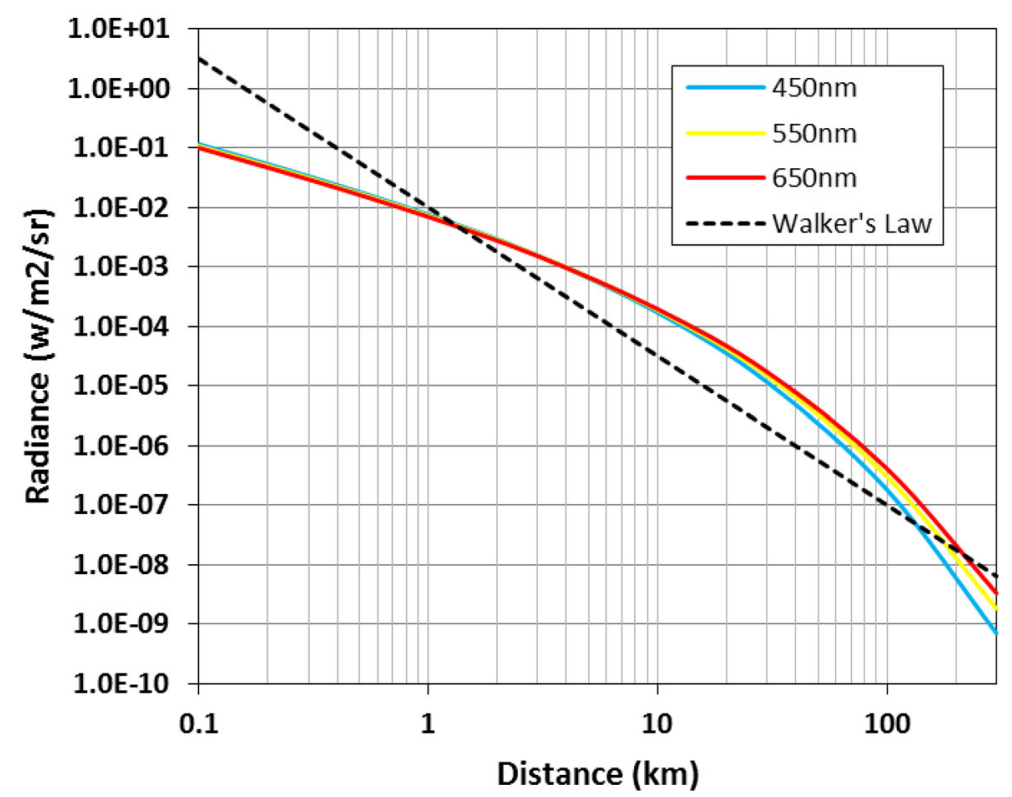

Fig. 3 Artificial (zenith) radiance versus distance predicted by a Garstang model, for three monochromatic sources of indicated wavelengths. The relation proposed by Walker is shown as the dashed line. Spectral issues are discussed in the following subsection

emissions, with the greater ratios observed at greater distance from the light sources. When used to assess lighting design issues such as the use of lighting fixtures with $1 \%$ direct upward emission, ${ }^{3}$ Luginbuhl et al. (2009a) found that artificial sky

\footnotetext{
3 Such a scenario has been proposed by roadway lighting designers, who suggest that allowing a small fraction of direct upward emission can allow the use of lighting fixtures with wider horizontal
} 
brightness will be increased between 30 and $100 \%$ at 50 and $200 \mathrm{~km}$, respectively, compared to that expected if a fully shielded fixture were used.

\subsection{Sky brightness versus distance}

The effect of a given light source on sky brightness increases rapidly with decreasing distance, proportional approximately to the inverse 2.5 power of the distance. This relation is dominated by a simple inverse square law modified by extinction and atmospheric curvature. The relation shown by a Garstang model, assuming a uniform atmosphere and curved-earth geometry, is shown in Fig. 3.

The implications of this relation for impacts on observatory sites are clear and have been long recognized: observatories and light sources should be located as far from each other as possible, but most critically, large light sources (e.g., sports facilities or large commercial/industrial developments) must not be located near observatories. A light source located at $30 \mathrm{~km}$ with a given impact on sky brightness will have an impact 15 times greater at $10 \mathrm{~km}, 135$ times greater at $3 \mathrm{~km}$, and over 650 times greater at $1 \mathrm{~km}$. Some lighting codes address this issue through so-called "lumen density" standards (e.g. lumens/acre or lumens/hectare as applied to entire development projects, or lumens $/ \mathrm{m}^{2}$ as typically applied to specific uses such as car parks), but these standards can effectively address impacts at observatory sites only when combined with land-use policies that define total areas and locations to which the area-specific standards are applied.

\subsection{Sky brightness versus lamp spectrum}

The effect of the spectral content of the artificial light source on sky brightness is of much recent interest spurred by the advent of white LED sources in outdoor lighting applications. While the consequences for astronomy can indeed be dramatic, the nature and causes of the increased impacts are commonly misunderstood.

The amount of scattering undergone by light in the atmosphere is wavelength dependent, with shorter wavelengths scattering more strongly. In the case of the molecular component of the atmosphere, the process is commonly called Rayleigh scattering and is proportional to $\lambda^{-4}$. For typical aerosols found in the atmosphere, the process is commonly called Mie scattering and is proportional to about $\lambda^{-1}$. Rayleigh scattering occurs approximately equally (within a factor of two) into all directions relative to its the incident beam, while Mie scattering is heavily weighted in the forward direction.

Increased scattering also means increased extinction as the term is used in astronomical contexts-most extinction in clear atmospheres arises from scattering rather than true absorption. Models show that when the observation point is located within about ten kilometers of the light source, the two effects approximately balance (Luginbuhl et al. 2014), as shown in Fig. 3. At greater distances, extinction 


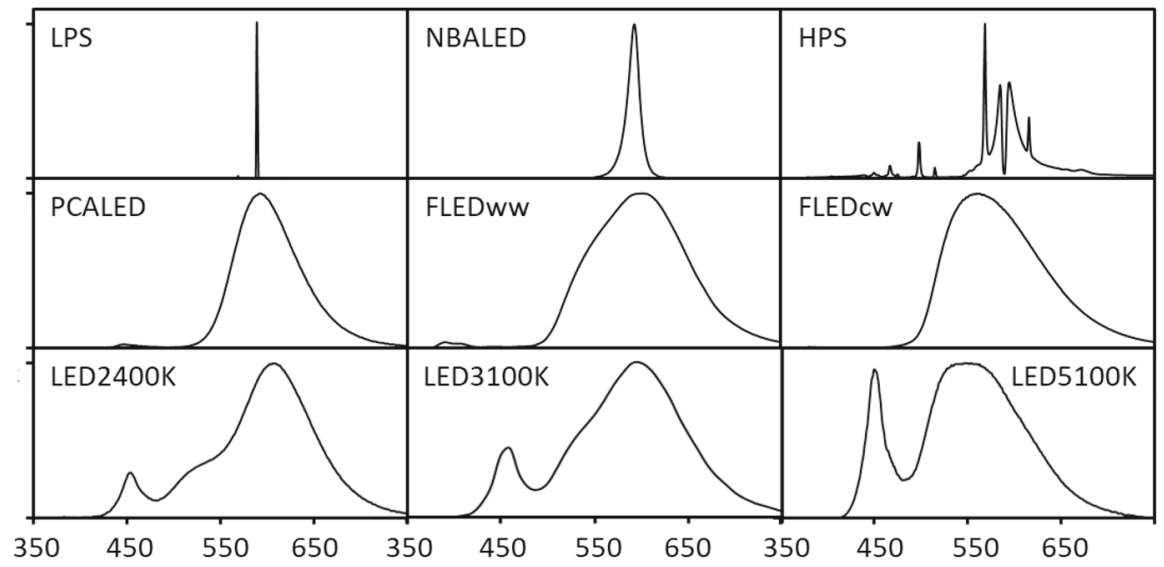

Fig. 4 Nine lamp sources evaluated for astronomical impacts: low-pressure sodium (LPS); "narrowband" or "direct" amber LED with peak emission at $595 \mathrm{~nm}$ (NBALED) (narrow-band amber LEDs can have peak wavelengths over a wide range. Though significantly redder than LPS or an NBALED peaking at $589 \mathrm{~nm}$, the spectrum analyzed here is representative of products offered by some manufacturers.); high-pressure sodium (HPS); phosphor-converted amber LED (PCALED); $500 \mathrm{~nm}$ filtered warm-white LED (FLEDww); $500 \mathrm{~nm}$ filtered cool-white LED (FLEDcw); white LEDs with correlated color temperature (CCT) of $2400 \mathrm{~K}, 3100 \mathrm{~K}$ and $5100 \mathrm{~K}$. For convenience, all spectra are normalized for the same peak intensity
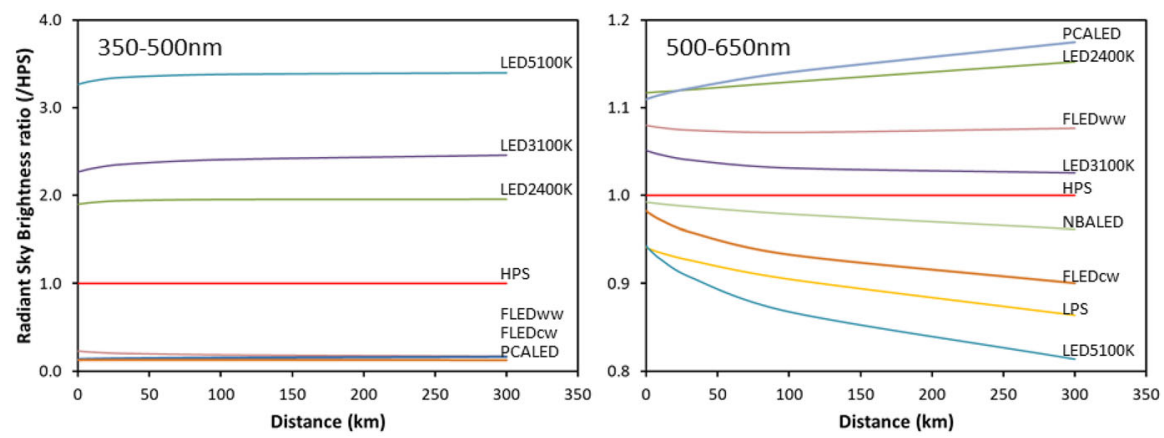

Fig. 5 Overhead radiant sky glow ratio as a function of distance. The left panel, relative to HPS, is for $\lambda 350-500 \mathrm{~nm}$ (LPS and NBALED have no emission here); the right panel is for $\lambda 500-650 \mathrm{~nm}$. All sources are balanced for equal luminous output. (Adapted from Luginbuhl et al. 2012)

begins to dominate, removing increasing proportions of the short wavelength emission and causing light sources, like the sun at sunset, to "redden."

The consequence of these processes from the professional astronomical perspective is mixed. Broad-spectrum or white light sources, for a given luminous flux, will have decreased flux in the yellow portion of the spectrum compared to yellow sources like HPS or LPS (or their LED analogs PCALED and NBALED). Thus radiant light pollution in the yellow portion of the spectrum will decrease under an HPS to LED conversion even in the equal-lumen condition. This is offset 
Table 1 Spectral bandwidth and relative scotopic to photopic ratio impacts of the nine lamp types shown in Fig. 4

\begin{tabular}{lllll}
\hline Lamp type* & Spectral bandwidth** $(\mathrm{nm})$ & \multicolumn{3}{l}{ Relative scotopic ratio*** } \\
\cline { 3 - 5 } & & Lamp & $1 \mathrm{~km}$ & $100 \mathrm{~km}$ \\
\hline LPS & 1 & 0.4 & 0.4 & 0.5 \\
NBALED**** & 34 & 0.5 & 0.5 & 0.6 \\
HPS & $62+$ & 1.0 & 1.0 & 1.0 \\
PCALED & 152 & 0.8 & 0.8 & 0.9 \\
LED 2400 K & 272 & 1.8 & 1.8 & 1.6 \\
LED 3100 K & 280 & 2.3 & 2.3 & 2.0 \\
LED 5100 K & 250 & 3.3 & 3.2 & 2.8 \\
FLEDww & 210 & 1.5 & 1.5 & 1.5 \\
FLEDcw & 211 & 1.9 & 1.9 & 1.9 \\
\hline
\end{tabular}

${ }^{*}$ Characteristics of LEDs, including NBALED, can be expected to vary depending on manufacturer and lot

${ }^{* * *}$ Wavelength range where radiance $>=10 \%$ of peak; + indicates discontinuous ranges

${ }^{* * * *}$ Relative to HPS with $\mathrm{S} / \mathrm{P}=0.64$

${ }^{* * * * *}$ Peak wavelength $595 \mathrm{~nm}$

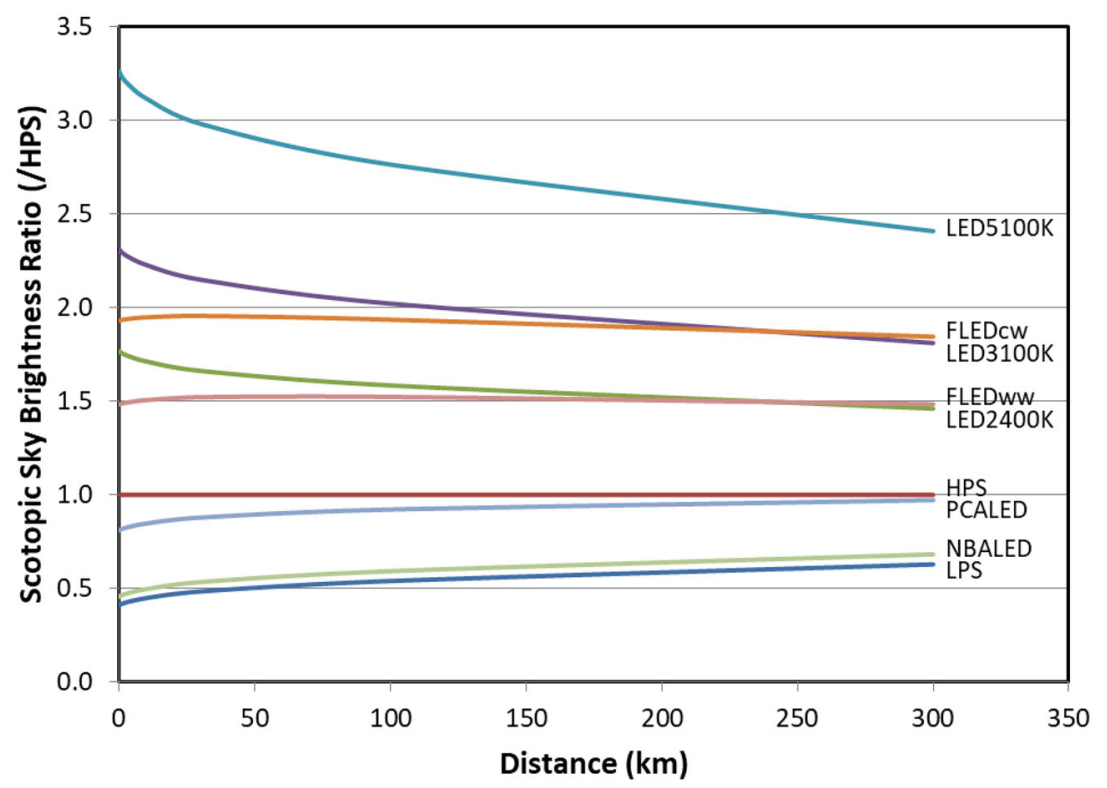

Fig. 6 Scotopic sky brightness ratio (relative to HPS) for the lamp types shown, as a function of distance. (Adapted from Luginbuhl et al. 2014) 
by a much more dramatic increase in the blue portion of the spectrum, where yellow sources have little or no emission.

To assess impacts arising from specific lamp types, nine lamp types representative of the range used in outdoor lighting are evaluated to illustrate the range of effects. The spectra of these lamps are illustrated in Fig. 4.

First examining impacts on professional astronomical observation, Luginbuhl et al. (2012) examined the radiant sky brightness integrated over wavelength ranges between $\lambda 350$ and $500 \mathrm{~nm}$ and $\lambda 500-650 \mathrm{~nm}$. Compared to predominant HPS, white LEDs increase radiance in the blue portion of the spectrum by factors of $\sim 2$ or more (Fig. 5, left). The filtered white LEDs offered by one fixture manufacturer substantially reduce radiant flux in this portion of the spectrum. In the red portion of the spectrum (Fig. 5, right) the filtered and white LEDs of CCT $3100 \mathrm{~K}$ and less increase radiant flux by smaller factors, ranging from 5 to $12 \%$ compared to HPS when observed from $10 \mathrm{~km}$ or less. The $5100 \mathrm{~K}$ LED has approximately a $5 \%$ lower radiance nearby, due to the decreased radiant flux of this lamp at wavelengths $>500 \mathrm{~nm}$. Due to the reddening effects of long path length through the atmosphere, the impacts shown here vary with distance, decreasing at larger distances for lamps with greater short wavelength emission compared to HPS.

The second metric useful to assess impacts on professional astronomical observation is the total emission bandwidth. Table 1 shows the spectral emission coverage for the nine lamps in Fig. 4.

The impact on visual astronomy is indicated by the $S / P$ or scotopic to photopic ratio, shown as a ratio to the $S / P$ of HPS in the last three columns of Table 1 and as a function of distance in Fig. 6. The three values listed in Table 1 are for the lamp, $1 \mathrm{~km}$ distance and $100 \mathrm{~km}$ distance. The bluer sources, including especially all of the white LEDs, exhibit significant reddening at the larger distance, though they remain of significantly greater $(1.6-2.8 \times)$ visual impact than the yellow sources even at $100 \mathrm{~km}$.

If an equal amount of lighting (lumens) is used for lighting systems utilizing these different lamp types, these ratios indicate the relative impact of the different lamp types on the visual brightness of the night sky. As an example, a lighting system using white LEDs with CCT of $3100 \mathrm{~K}$ instead of HPS (or alternatively replacing HPS with the same white LEDs) can be expected to have visual sky glow impacts (or to increase impacts) arising from this portion of the lighting ${ }^{4}$ of $2.3 \times$ or $130 \%$ greater nearby, and $2.0 \times$ or $100 \%$ greater at $100 \mathrm{~km}$. If considering a change from LPS to even the lowest CCT white LED $(2400 \mathrm{~K})$, the expected sky glow increase from the new lighting would be $1.8 / 0.5=3.6 \times$ or $260 \%$ nearby, and $1.6 /$ $0.5=3.2 \times$ or $220 \%$ at $100 \mathrm{~km}$.

Some lighting recommendations suggest that broad spectrum or blue-rich sources such as white LEDs can achieve equivalent performance to yellow sources such as HPS or LPS using lower (photopic) luminances, and thus lower total luminous output in the installation. The acceptance of this in the lighting design community, in the sense that lighting levels using broad-spectrum sources are actually being reduced, is not widespread. A more common outcome of improved efficiencies and

\footnotetext{
${ }^{4}$ The difference will be diluted if there is other lighting.
} 
perceived improvements in performance is to maintain or increase lighting levels during lighting retrofits or in new lighting installations. In some important applications such as roadway lighting, decreasing illumination levels with broadspectrum sources has been explicitly deprecated. Even so, the spectral adjustment factors often discussed are in the range of $30 \%$ or less, which for all of the blue-rich sources assessed here is insufficient to overcome the increased visual sky glow impact due to the increased $S / P$ ratio.

Another factor that can reduce impacts of LED systems compared to bulb-based technologies such as LPS or HPS is the possibility of lumen reductions through improved "target efficiency," that is, higher efficiency at directing light into the area that needs illumination. This advantage is particularly noticeable when the illuminated area is relatively constrained or narrow, such as for some roadways, and can amount to approximately $30 \%$ in some cases. Again, alone, this advantage is insufficient to offset the increased visual impact of all of the white sources assessed here. This efficiency advantage applies equally to the low-impact yellow LED sources such as PCALED and NBALED, providing the opportunity to reap the sky glow benefits of both yellow light and reduced luminous flux.

It is important to note that for visual observers, the dominant effect behind increased artificial sky brightness with white (e.g. LED) light sources arises from the increased sensitivity of the dark-adapted eye to blue and green light (i.e. the Purkinje shift), and not as commonly claimed, from Rayleigh scattering of short wavelengths. As can be seen in Fig. 3, the increased scattering is approximately balanced by increased extinction for distances nearby-at distances greater than about $10 \mathrm{~km}$ the increased extinction dominates and white light sources of a given luminous output will have less impact on radiant sky glow. Nonetheless, the dramatically increased sensitivity of the dark-adapted human eye to blue and green lights wavelengths means that even at greater distances white light sources have greater visual impact than yellow sources such as HPS, LPS, PCALED or NBALED.

\section{Field measurements of artificial night sky brightness}

The "quality" of the night sky refers to how closely it matches a natural photic condition, a natural lightscape. In a simplistic ideal model, a perfect (terrestrial) view of the heavens is achieved when there are no interfering artificial sources visible to the observer and interference from the Earth's atmosphere is minimal; astronomical observatories are located on high mountaintops to avoid its blurring and extinction effects on astronomical light sources. Since 2006, the mission of the U.S. National Park Service (NPS) is to preserve natural scenery and the natural photic environment (NPS 2006). In this context, the natural effects of the atmosphere are not considered as degrading sky quality per se. Therefore, the assessments of the U.S. NPS of sky quality are limited to the detection and quantification of anthropogenic or artificial light in the nighttime environment. 


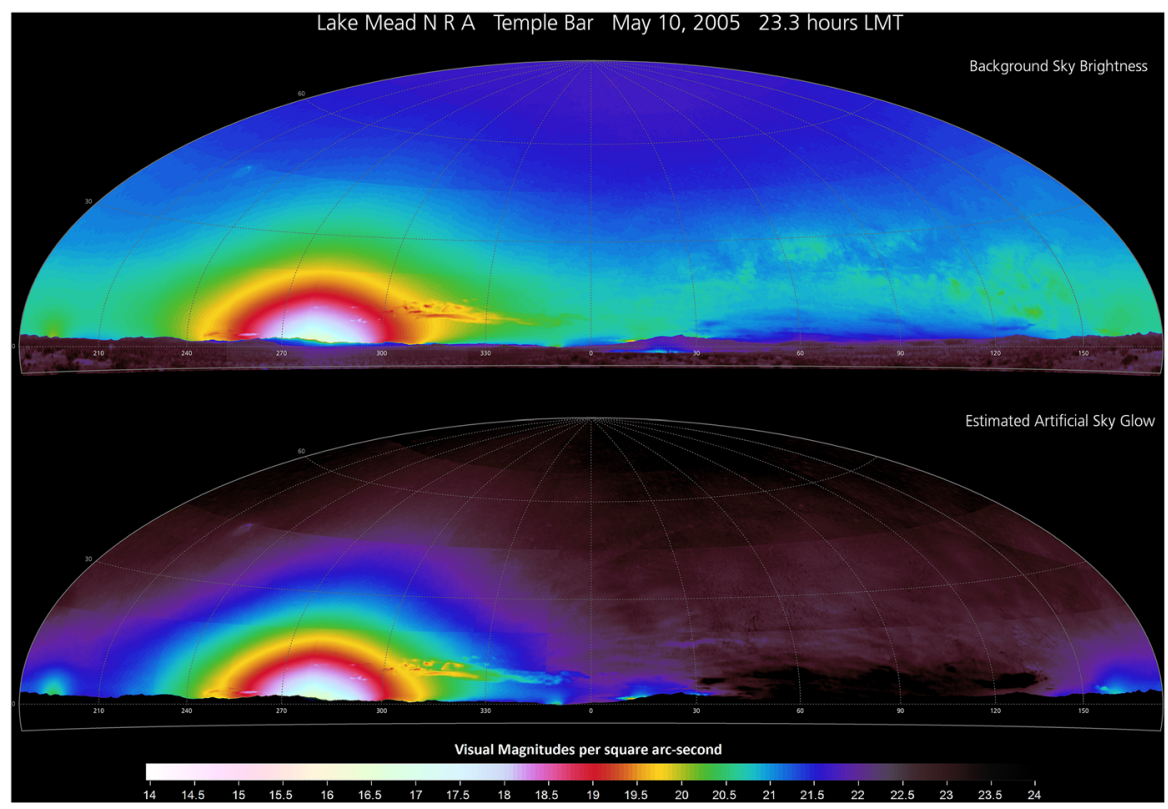

Fig. 7 Upper: all-sky image of Lake Mead with artificial sky glow contribution of Las Vegas on the horizon, taken and processed by author DD. This image records total sky glow with both artificial and natural sources. Lower: all-sky image with modeled natural sky glow contribution subtracted

One of the most interesting features of the night sky is the variation observed in natural sky brightness from night to night, or even hour to hour on the same night. Any attempt to quantify artificial sky glow must necessarily remove the natural background from the observed data, variable as it may be. In NPS work, a customized natural sky brightness model is developed for each data set at a resolution of 0.05 degrees (Duriscoe 2013). The features of the Milky Way and the Zodiacal Light are registered with each frame and accurately removed. Figure 7 is an illustrative example. The natural airglow is the most challenging feature to accurately model and remove frame by frame and pixel by pixel. The airglow is always present in the night sky, but varies in intensity over periods of minutes and may have variations over the whole sky (Roach and Gordon 1973). Errors in either over- or under-subtraction of this feature have been found to occur under such conditions, typically with a maximum of $20 \%$ of the average natural background.

\subsection{Quantitative sky quality indicators}

An all-sky image of estimated artificial sky glow may then be used as the primary source of data for computing numeric indicators of visual sky quality. A powerful tool is the distribution of sky brightness values from each cell in the all sky image. The higher the resolution (greater number of cells), the closer the image distribution will be to the true distribution. It is important that each pixel in the image (or observation) represents an equal area of the sky, and that all areas of the sky are 
weighted equally. Figure 8, panels 1-6, shows how the distribution of sky glow measures varies as impacts from artificial sources increase. As a greater proportion of the sky falls above a certain brightness threshold, impacts to the natural lightscape become more severe. These thresholds may be defined in terms of human vision or the response of other organisms.

Indicators of sky quality derived from artificial all sky luminance maps are described by Duriscoe (2016). Here we focus on the use of the average of all observations throughout the sky, rank statistics performed on the luminance values, particularly the median or 50th percentile, and the "brightest square degree" or the 99.995th percentile, and the use of horizontal and maximum vertical illuminance is described.

The arithmetic mean of luminance measures or average artificial sky luminance is an excellent indicator for protected areas, where a measure of the overall visual experience is desired. Even if the zenith area is relatively free of light pollution, high values from bright light domes near the horizon will significantly contribute to the average, resulting in areas far from light pollution sources recording significant average numbers. This has the effect of increasing the scope of influence of large metropolitan areas upon the degradation of sky quality to areas up to $300 \mathrm{~km}$ distant.

Median artificial sky luminance often represents the most frequently occurring sky brightness throughout the sky. This indicator is of value in predicting the visual impact of sky glow, as it represents what a visual observer will most often see when looking up at the sky. By definition, half the sky is at least as dark as the median. Typically, the darkest half of the sky is that part which is closest to zenith, and onehalf the area of the hemisphere is included in the portion above zenith angle 60 degrees. Therefore, the median may be inferred to indicate the brightest sky luminance in the "upper one half" of the sky, a value of particular interest to amateur and professional astronomers.

The brightest square degree has an important effect on human vision. Above a certain threshold, the human eye will begin to lose dark adaptation. If a certain part of the sky, usually at the core of a city light dome near the horizon, is above this threshold, scotopic vision will be impaired. Therefore, this value may be used as an indicator of an observer's ability to appreciate a natural night environment. The area of sky encompassed by each pixel or the resolution of the all-sky image will affect the maximum value, especially at locations distant from large cities which typically exhibit relatively small light domes with a small bright core. High resolution imaging systems will record the maximum luminance as a brighter value than lower resolution devices in such situations. A system which produces at least one degree resolution is therefore necessary to derive this statistic. Since there are about 20,626 square degrees in the hemisphere, the brightest square degree corresponds to the rank statistic 20,625/20,626 or the 99.995th percentile.

Illuminance from artificial sky glow, horizontal or vertical, is a good indicator of the effect upon the human visual experience and the landscape of protected areas. Horizontal illuminance provides a general illumination to the land, while vertical illuminance creates shadows. Bright light domes from distant cities may produce a strong vertical illuminance in an otherwise relatively dark location. The natural 

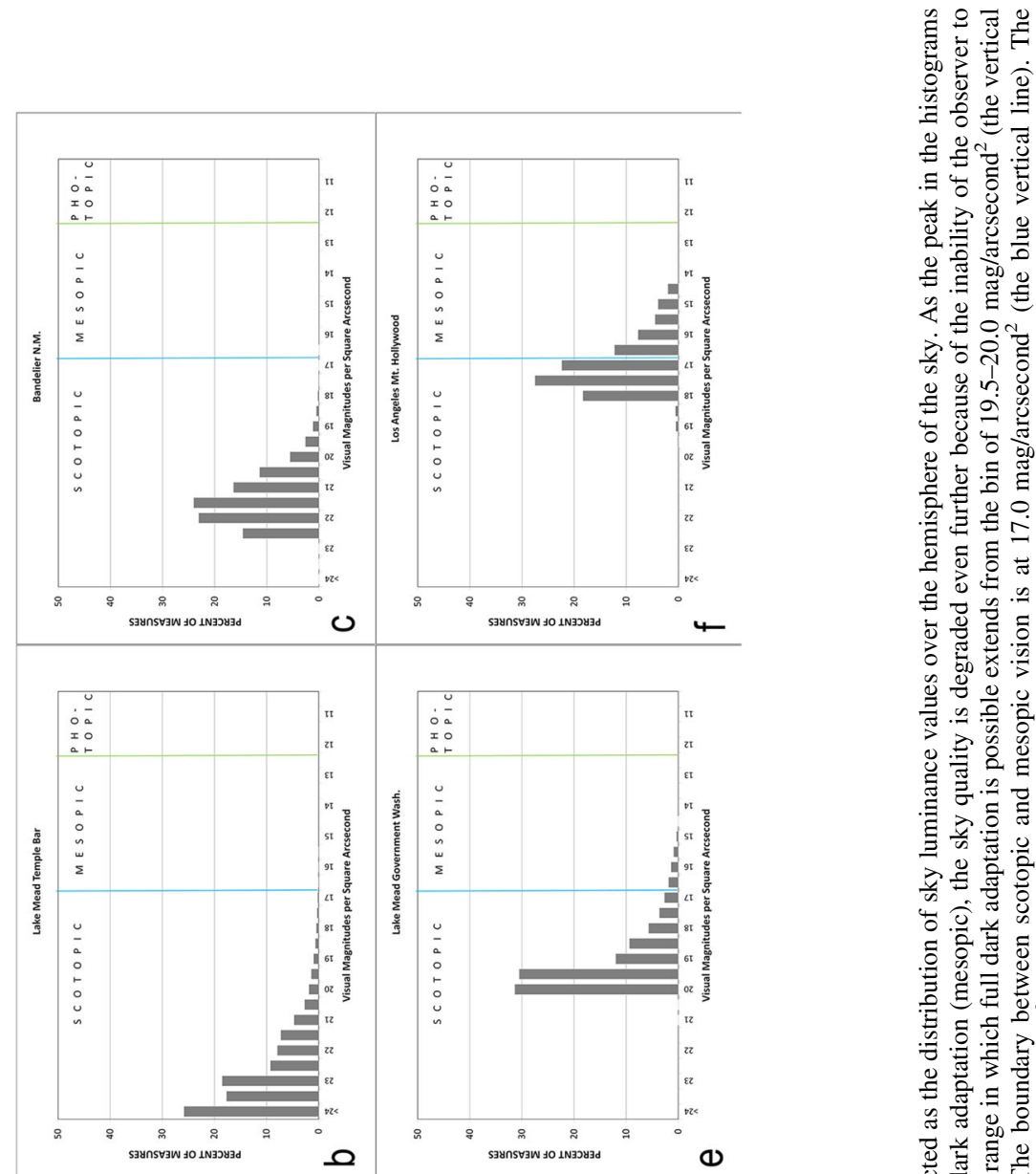

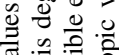

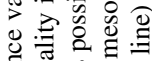

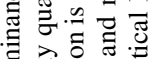

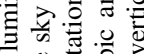

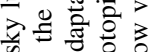

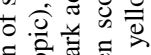

응 홍

丞芯

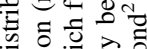

$\because$ 을

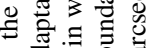

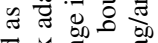

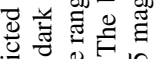

证

包

3宅司

on $=0$

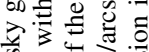

क 0 क्ष

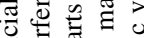

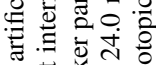

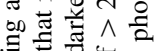

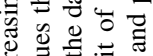

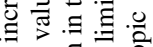

पू छ

‡ 寻灵

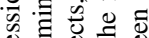

品三造气

는 긍

《亭焉离

$\infty \stackrel{0}{0}$

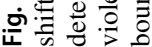


reference condition for these indicators is predicted at 0.8 mlux for horizontal, 0.4 mlux for vertical (Duriscoe 2016). All of the indicators may be expressed as a ratio to natural conditions, or "percent above natural."

From a decomposition of the night sky spectrum, Kollath et al. (2020) determined that the 'continuous' component of the natural sky (zodiacal light, scattered starlight and airglow pseudo-continuum) is nearly constant at all visible wavelengths and has a spectral radiance of $\sim 2 \mathrm{nW} \mathrm{m}^{-2} \mathrm{sr}^{-1} \mathrm{~nm}^{-1}$, or $2 \mathrm{dsu}$ (dark sky units). Because of the relatively limited range of broad-band color variations of natural skyglow under clear, moonless conditions, digital camera-based, three-color (RGB) radiance measurements in dsu give a usable sky brightness measurement (Kolláth and Kolláth 2020). Use of a uniform and calibrated index, such as dsu was a recommendation of the D\&QS Report for professional observatories (Walker et al. 2020b).

The National Park Service Sky Quality Index predicts the impact of sky glow on the visual scene of the night sky as perceived by the fully dark-adapted human eye. It is a synthetic index made up of four components, (1) the percentage of the night sky that contains no artificial sky glow and therefore is available to the observer, (2) the impact of the areas of the sky that contain sky glow upon the observer's scotopic vision, or ability to dark adapt, (3) the effect of sky glow on the contrast between the Milky Way and the sky background, and (4) the effect of sky glow upon the ability of the observer to see faint stars, specifically, the reduction in number of faint stars over the entire sky. Each component is weighted equally. It is based entirely upon data from the artificial sky glow all sky image; the index cannot be calculated from raw sky brightness measurements. It is insensitive to atmospheric transparency and seeing, as well as the amount of natural airglow in the sky, which is presumed to be removed before analysis.

The index is subtractive; varying levels of artificial sky glow lead to subtraction from a possible score of 100, 25 in each of the four components. A score of zero indicates that all semblance of the natural night sky is lost, and, even though a few bright stars may be visible, the observer has no opportunity to dark adapt, the Milky Way is totally invisible, and no area of the sky is free of light pollution. The component "available natural sky" is very sensitive to small amounts of sky glow. All areas of the sky are weighted the same, hence small light domes along the horizon exact heavy penalties from that component; the index decreases rapidly from 100 as sky glow increases. Scores of 80-85 are common for sites which many observers consider excellent.

Figure 9 illustrates the sensitivity of the four components to increasing sky brightness. Each pixel in the artificial sky glow image is placed into 0.5 mag $\operatorname{arcsec}^{-2}$ bins, and each bin is assigned a weighting factor based upon the brightness value. The weighting factor is multiplied by the percentage of pixels in the bin, and the products are summed for each of the four components. A grand sum of the components is then subtracted from 100 to yield the index.

Figure 10a-d shows simulations of the effect of increasing amounts of sky glow from a city in the direction of the observer toward the bottom left of the illustrations. Details in the Milky Way and the number of stars visible to the unaided eye are progressively degraded, two components of the index. Figure 10a represents a sky 


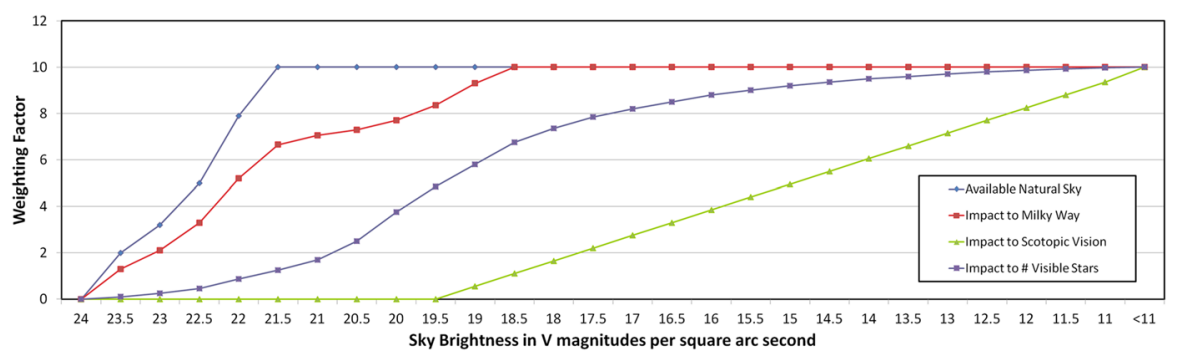

Fig. 9 Graph showing the relative sensitivity of each of the four components of the sky quality index as the background artificial sky brightness increases. At 24 mag $\operatorname{arcsec}^{-2}$ and fainter, the night sky view is considered to be pristine, while at $10.5 \mathrm{mag} \operatorname{arcsec}^{-2}$ and brighter it is predicted that all components contributing to the ability to observe the night sky are totally lost

with no artificial sky glow, and would receive an SQI of 100. Figure 10d illustrates a location where the Milky Way may not be detected, the artificial sky glow is at least as bright as 20.5 mags $\operatorname{arcsec}^{-2}$, the number of visible stars is severely reduced, and the impact to scotopic vision is significant.

\subsection{Examples}

Selected sites including from observatories and protected areas are given to illustrate the use of the all sky artificial sky glow image to predict impacts to astronomical observations and visual sky quality. Table 2 lists the sites and indicators for each described above, sorted on the sky quality index from highest to lowest. Figure 11 a-m shows the artificial sky brightness in a fisheye projection of the hemisphere color coded in $\mathrm{V}$ mags $\operatorname{arcsec}^{-2}$.

From Table 2 and Fig. 11 it can be seen that at sites a-d, while the artificial zenith sky brightness remains below $10 \%$ above natural and essentially cannot be measured, the sky quality index decreases from 99.3 to 87.8 and the median artificial sky brightness increases to $17.8 \%$ above natural. In addition, the artificial component of the brightest square degree at site $d$ is measured at 17.6 mag arcsec $^{-2}$, bright enough to inhibit dark adaptation of the human eye. Site $d$ in Death Valley National Park, California is more than $100 \mathrm{~km}$ from Las Vegas, Nevada, city center but the sky is still bright enough to significantly degrade visual sky quality. Site e, near Flagstaff, Arizona, exhibits measurable zenith sky glow but the brightest square degree is actually significantly dimmer than site d. This pattern demonstrates that a large city (Las Vegas) at greater distance may produce much brighter sky glow near the horizon than a smaller city (Flagstaff) which is much closer to the observer.

Sites f-k all have the brightest square degree at levels that inhibit dark adaptation, and the median artificial sky glow ranges from 48.6 to $250 \%$ above natural. These sites would probably not be suitable for astronomical research in the visual range of optical wavelengths. Yet amateur astronomy groups regularly visit several of these sites, considering them to be "dark sky" observing sites near large metropolitan areas. Sites 1 and $\mathrm{m}$ represent locations at the margin of and within 


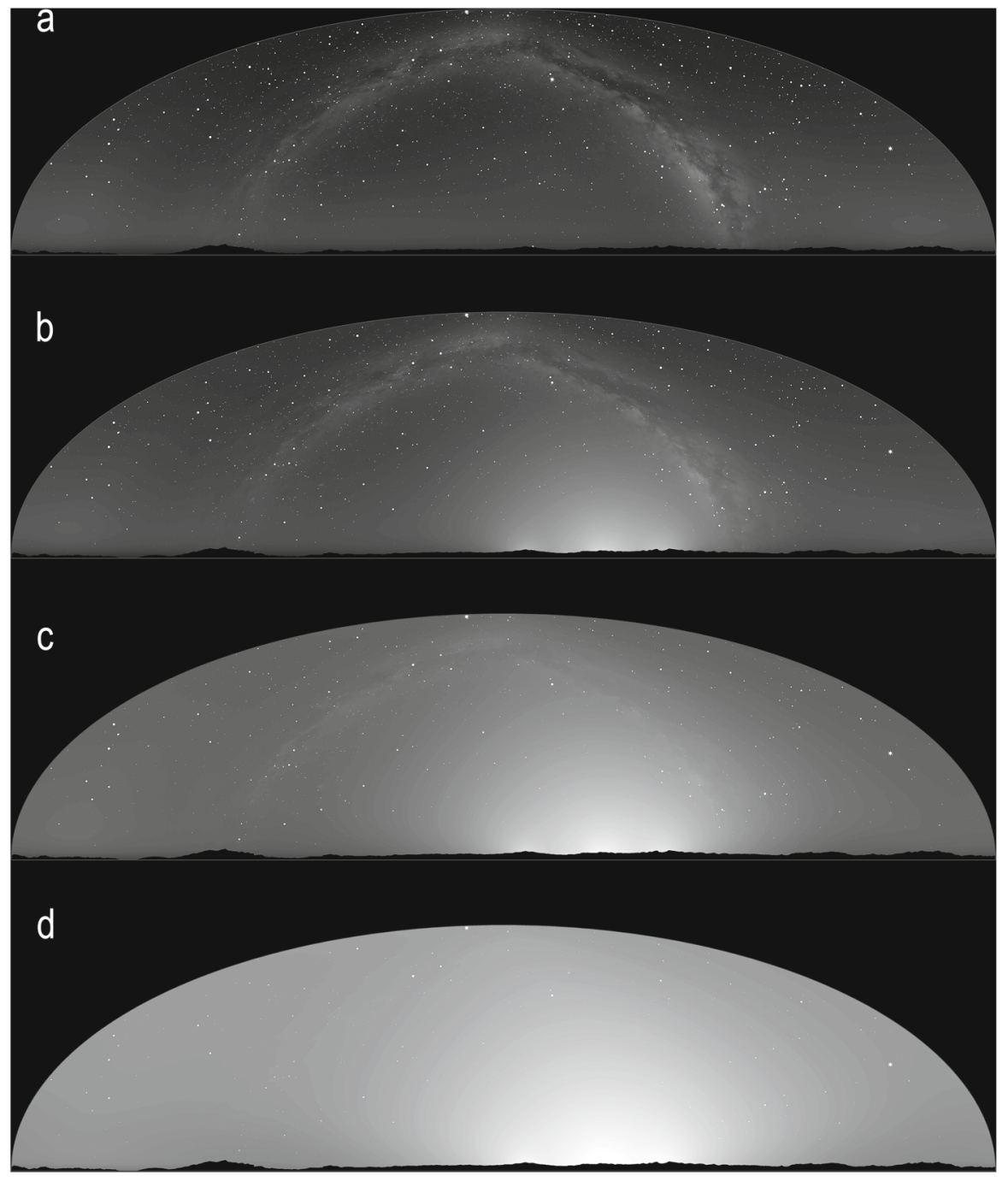

Fig. 10 a-d Illustrations of the effect on visual features of the night sky of increasing artificial sky glow. The sub-figures a, b, c, and d represent 0, 1, 6, and 36 times the amount of light at the source, respectively. The city is Page, Arizona modeled from VIIRS DNB upward radiance data as viewed from $15 \mathrm{~km}$ distant. The simulated stars are progressively extinguished according to an algorithm that predicts whether or not a star of a particular brightness will be visible to the unaided eye given its immediate background brightness

large metropolitan areas (Las Vegas, Nevada, and Washington, D.C., respectively), where the artificial sky glow is thousands of percent above the natural levels for all of the indicators, and dark adaptation is impossible. 


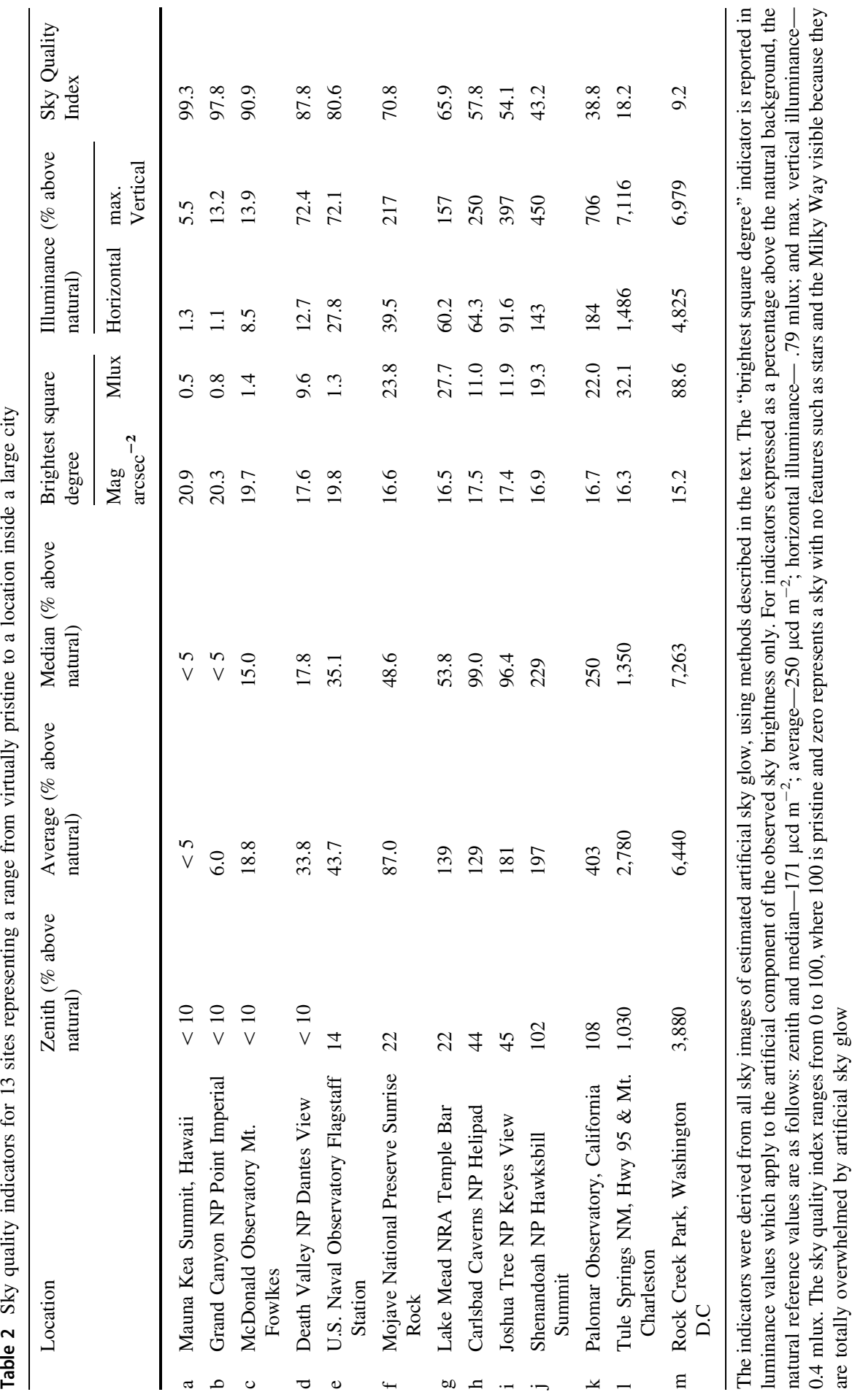


(a)
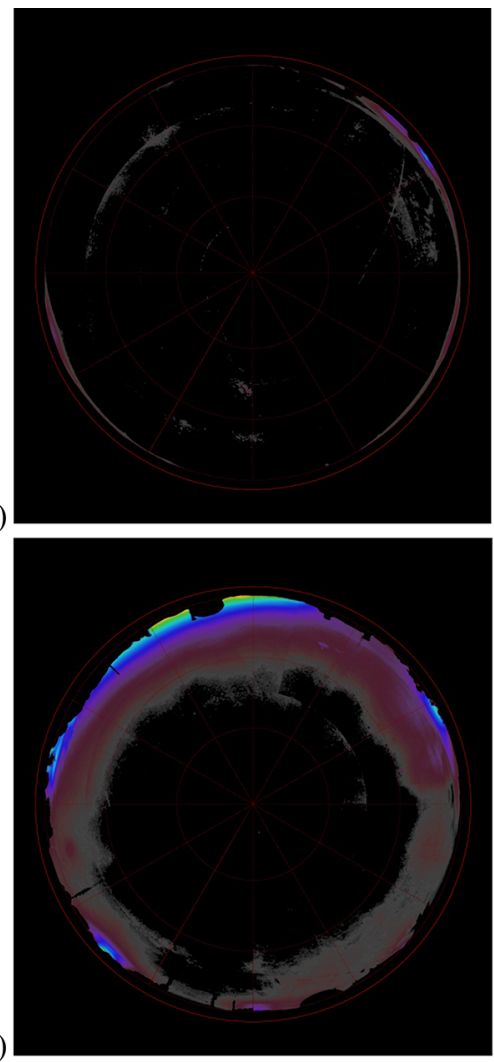

(c)

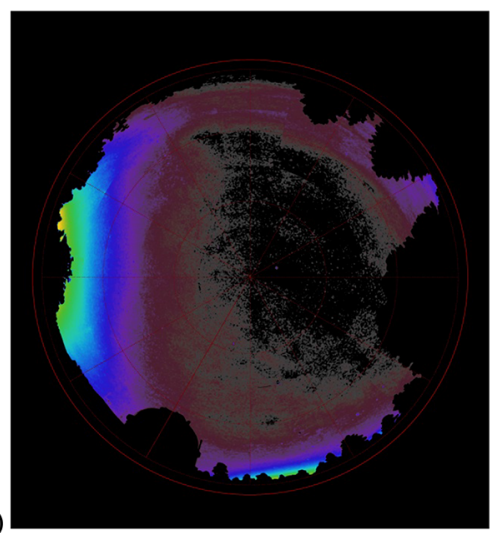

(e)

(f)

(b)

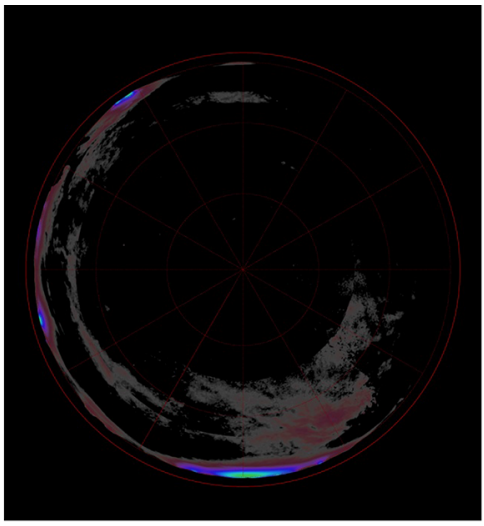

(d)
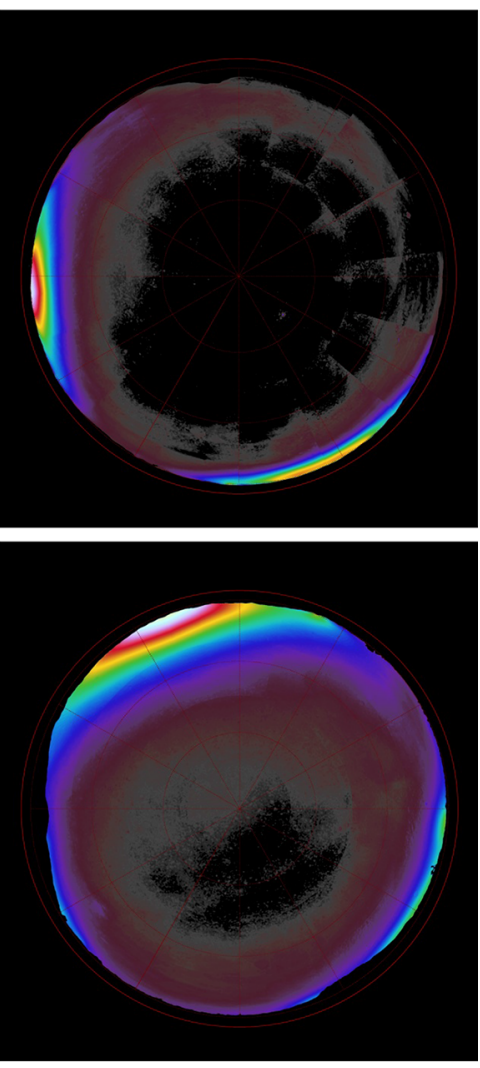

Fig. 11 a-n Panels (a) through (m) are illustrations of the all-sky estimated artificial sky glow in false color measured in $\mathrm{V}$ mag $\operatorname{arcsec}^{-2}$ for the 13 sites described in Table 2; panel (n) is the color map legend. For illustrations (a) through (e), dark gray represents approximately $23.7 \mathrm{mag} \operatorname{arcsec}^{-2}$ which is $21 \%$ above the natural background. Therefore, significant ( $>10 \%$ above natural) sky glow may be present even in the black areas 
(g)

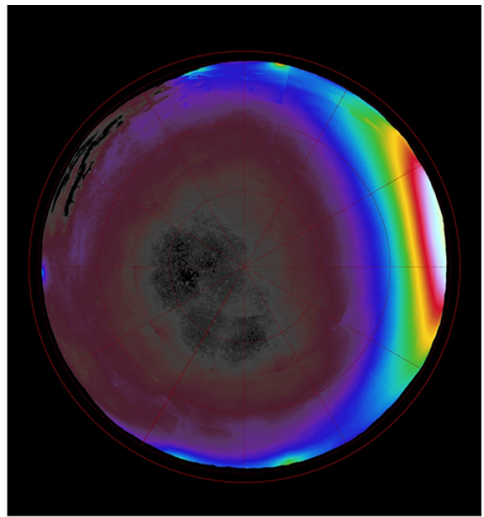

(i)

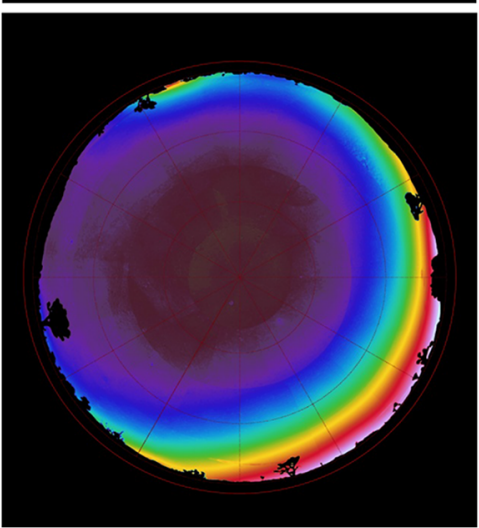

(k)

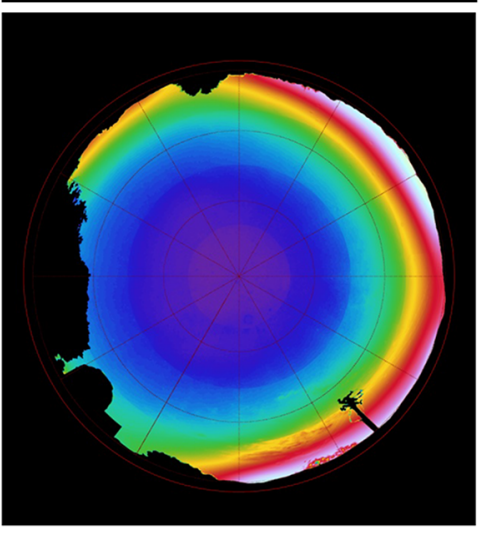

(h)

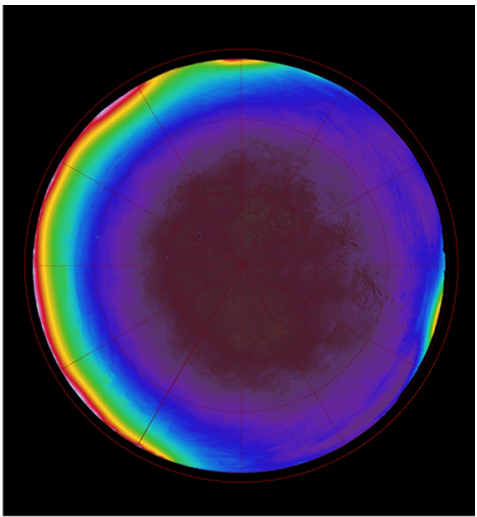

(j)
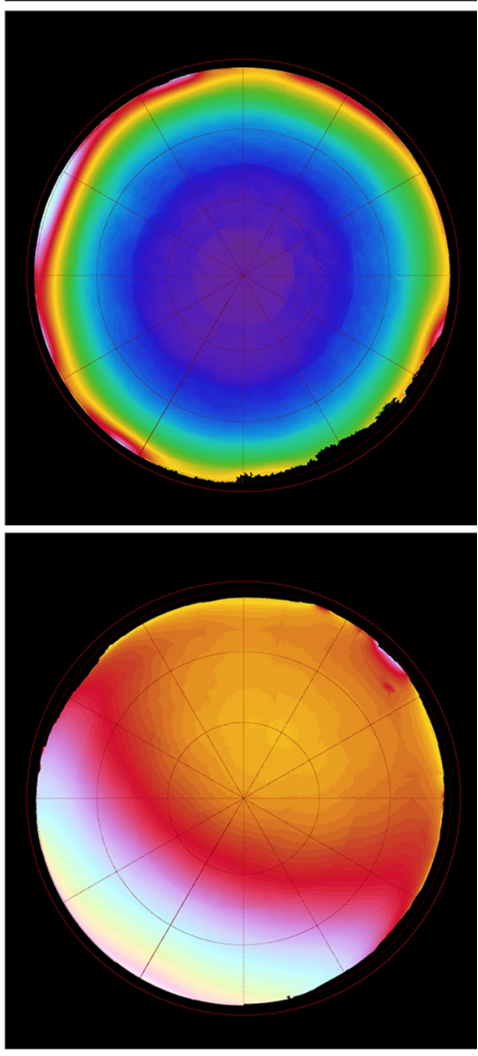

Fig. 11 continued

\section{Sky brightness measurements and impact of artificial sources}

With few exceptions, growing sky glow from artificial sources negatively impacts the sky background recorded at major observatories around the world. Increasing urbanization and nighttime illumination of those urban centers create a spreading 


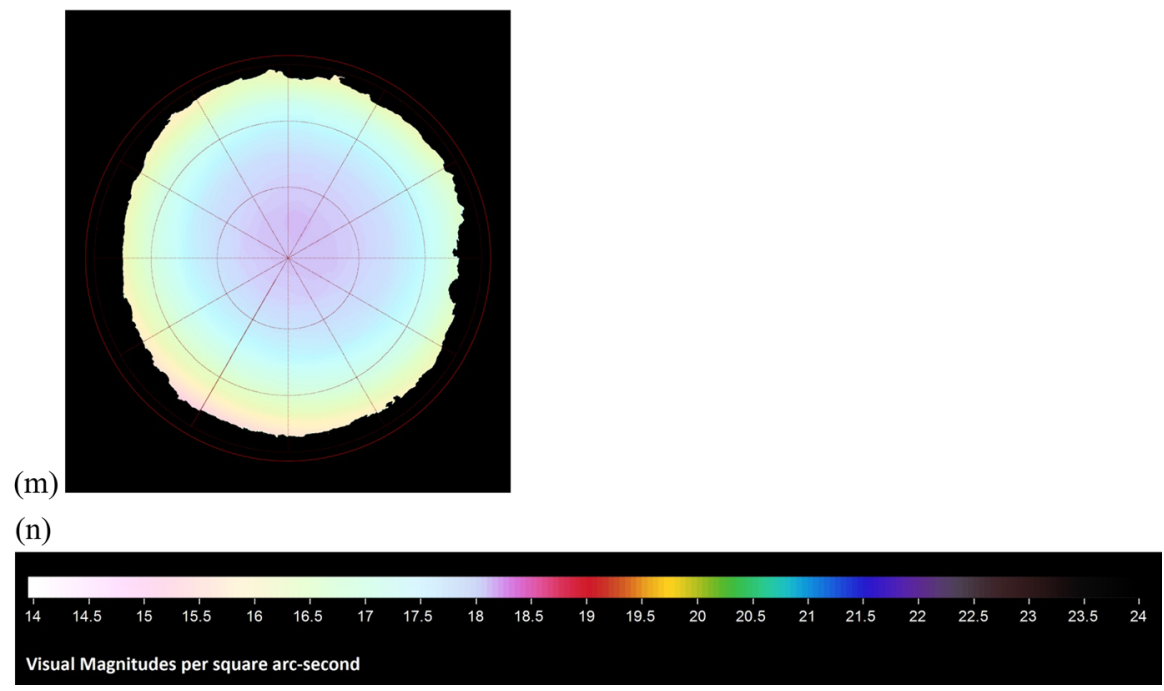

Fig. 11 continued

Table 3 Sky Brightness (mag/ $\operatorname{arcsec}^{2}$ ) vs. lunar age

\begin{tabular}{llllll}
\hline Lunar age (days) & $\mathrm{U}$ & $\mathrm{B}$ & $\mathrm{V}$ & $\mathrm{R}$ & $\mathrm{I}$ \\
\hline 0 & 22.0 & 22.7 & 21.8 & 20.9 & 19.9 \\
3 & 21.5 & 22.4 & 21.7 & 20.8 & 19.9 \\
7 & 19.9 & 21.6 & 21.4 & 20.6 & 19.7 \\
10 & 18.5 & 20.7 & 20.7 & 20.3 & 19.5 \\
14 & 17.0 & 19.5 & 20.0 & 19.9 & 19.2 \\
\hline
\end{tabular}

area of scattered artificial light, impacting observatories that were established when such conurbations were much smaller and much less brightly illuminated. In more remote areas, mining activities and rural electrification move sky brightness levels off the floor of zero contamination.

In the first instance, assessment of impact requires a reliable baseline of sky background measurement, for which multiple major observatories have published their results. A particularly thorough review and analysis was performed by Benn and Ellison (2007), based on ten years of monitoring of the night sky brightness over La Palma. Their Table 1 has a comparison of night sky surface brightness measurements in standard broad-band magnitudes for multiple observatory and potential sites published from 1970 through 1997. Their list included only those sites where the impact from light pollution was modeled to be less than $0.1 \mathrm{mag}$, so excluded Calar Alto, Lick, Lowell, Mt. Wilson and Palomar. The published observations were taken at ecliptic latitude $>40^{\circ}$, so that variations in zodiacal light contribution were likely to be $<0.1 \mathrm{mag}$. A consistent result for dark of the moon and high Galactic latitudes was that such a dark sky resulted in a $\mathrm{V}$ surface 
brightness of $21.9 \mathrm{mag} \operatorname{arcsec}^{-2}$ and corresponding B of $22.9 \mathrm{mag} \operatorname{arcsec}^{-2}$. A similar table including Calar Alto was published by Sanchez et al. (2007).

The strongest variation in optical light night sky brightness is driven by lunar phase. Typical variations are summarized in the Kitt Peak National Observatory compilation of measurements from A. Walker (1987) reproduced in Table 3.

The greatest variation is at the shortest wavelengths from the Rayleigh scattering of reflected sunlight. When high-pressure sodium lamps were in common use, those wavelengths tended to be less contaminated by artificial sky glow. The visual and longer wavelength bands still brighten by quarter moon such that only the darkest part of the month is measurably affected by artificial sky glow at sites where that contribution is $10 \%$ or below. A valuable listing of other night sky brightness levels is provided by Hänel et al. (2018).

A key point to note is that solar activity drives the airglow contribution to vary during the 11-year solar cycle. Based particularly on data by Krisciunas (1997) and Krisciunas et al. (2007), as well as other long-cycle measurements, Benn and Ellison (2007) and Walker and Schwarz (2007) conclude that the mean V zenith sky brightness at new moon brightens by $\sim 0.5$ mag $\operatorname{arcsec}^{-2}$ from solar minimum to solar maximum. The airglow variation corresponds directly to the $10.7 \mathrm{~cm}$ solar radio flux variation, with the highest correlation found for the radio flux measured 4.5 days prior to the zenith sky measurement (Krisciunas et al. 2007). The small auroral contribution can lag by some 2 years (Roach and Gordon 1973). The most recent solar minimum was in 2019.

Sky brightness monitoring for professional observatories continues to be reported. Neugent and Massey (2010) compared spectrophotometry for Kitt Peak over a decade interval, with azimuthal sampling to gauge the impact of urban growth. Patat (2008) reported 6 years of monitoring for Cerro Paranal. Zhang et al. (2015) as well as Yao et al. (2012) describe conditions at Xinglong Station outside of Beijing, the site of the LAMOST telescope, with a light dome that brightens the zenith by almost a magnitude with strong azimuthal variation. Aubé et al. (2014) characterize the sky brightness at two interior Argentinian sites, LEO ++ and El Leoncito. Riddle et al. (2008) predicted the impact of measured artificial sources on the horizon at prospective TMT sites, while Müller et al. (2011) report a trial sky measurement on Cerro Armazones. Further exploration of future or developmental sites is reported by Hampf et al. (2011) for southern Australia and by Zou and Zhou (2011) for Dome A in Antarctica. Pedani (2009) measured the sky brightness from Mt. Graham in southeastern Arizona, site of the Large Binocular Telescope. The zenith sky brightness is $\sim 0.1$ mag brighter than the dark sky limit, and in clear conditions that brightness increased by an additional 0.1 mag at $45^{\circ}$ zenith distance in azimuthal directions toward the two urban light domes. An important note of his measurement is that thin cloud could increase that contribution by up to 0.5 mag from forward scattering. Pedani (2009) noted general agreement with the earlier work by Taylor et al. (2004), whose 'darkest' sky brightness values were quoted by Sanchez et al. (2007). We note that the Taylor et al. measurements were taken near the peak of Solar Cycle 23, while Pedani's were near the end of that cycle. Krisciunas et al. (2010) found that an increase in brightness near horizon from artificial sources did not lead to a measurable increase in zenith brightness in the 
context of a Garstang (1989a) model. Luginbuhl et al. (2009b) found that the upward component of illumination from Flagstaff as measured at the U.S. Naval Observatory was lower than expected from the total light output and the Garstang model. They attributed the difference to additional shielding from structures and vegetation and presented a modified angular distribution function for a better fit to the measurements.

A site-by-site comparison of artificial sky glow contribution remains challenging in detail because of variations in airglow even at solar minimum, the stochastic contribution of faint stars through the various small apertures used for most of these measurements, and seasonal variation of aerosols in the atmosphere. Another approach is to use photometry from down-looking Earth-orbiting satellites, then calculate the scattered light impact at zenith for any point on the globe. Cinzano and Falchi (2012) describe in detail an extended Garstang (1989a) scattering model that they developed to be applied to such data. A natural limitation for a fully global application is that it applies the same mix of aerosol and molecular scattering at all points on the globe, so that the scattering impact on areas of good transparency typical for observatory sites is likely to be somewhat overestimated. For example, Cinzano and Falchi find a better fit to the Southwestern US with a scattering coefficient of half the value used more generally. However, there is no airglow or celestial component dominating the measurement, so actual deviations are likely to be only from the scattering assumptions. Detailed local conditions, such as topographical blocking, are not specifically modeled, and can produce significant overestimates of zenith sky brightness in particular cases.

In their remarkable paper, Falchi et al. (2016) analyze data from the Visible Infrared Radiometer Suite on the Suomi NPP weather satellite. The particular data stream used is from a scanning CCD with a broad-band filter, the "Day/Night Band", covering 0.5-0.9 $\mu \mathrm{m}$. They derived a best fitting upward radiation pattern with three major components, the strongest being a Lambertian. The atmospheric scattering model was computed specifically for the $\mathrm{V}$ band. The fit was carefully calibrated by worldwide data from up-looking sky quality measurements, corrected through the Duriscoe (2013) model of natural components in the V band to contain only the artificial light contribution. For construction of a final map, there is the implicit assumption that the spectrum of urban up-lighting is similar from place to place, both within the V-band of the component model and in the transformation from both the standard V-band and the Sky Quality Meter response in its $\sim 0.32$ $\mu \mathrm{m}-0.61 \mu \mathrm{m}$ band to the VIIRS DNB band of $0.5-0.9 \mu \mathrm{m}$. Zones near observatories where artificial lighting is dominated by low-pressure sodium or modern narrowband amber LED replacement with little metal halide in the mix will not in reality scatter as strongly as sources with stronger blue-green energy output. They adopted a zero-point of $V=22.0 \mathrm{mag} \operatorname{arcsec}^{-2}$, corresponding to $174 \mu \mathrm{cd} \mathrm{m}{ }^{-2}$ to compare the artificial light luminance at each point. They note that the typical satellite pass was at about 1:00 AM local time, when a fraction of the commercial lighting may have been turned down or off and vehicular traffic is significantly reduced (Luginbuhl et al. 2009a), so that the artificial brightness will be greater earlier in the night, which they parameterized by a fitting function. 


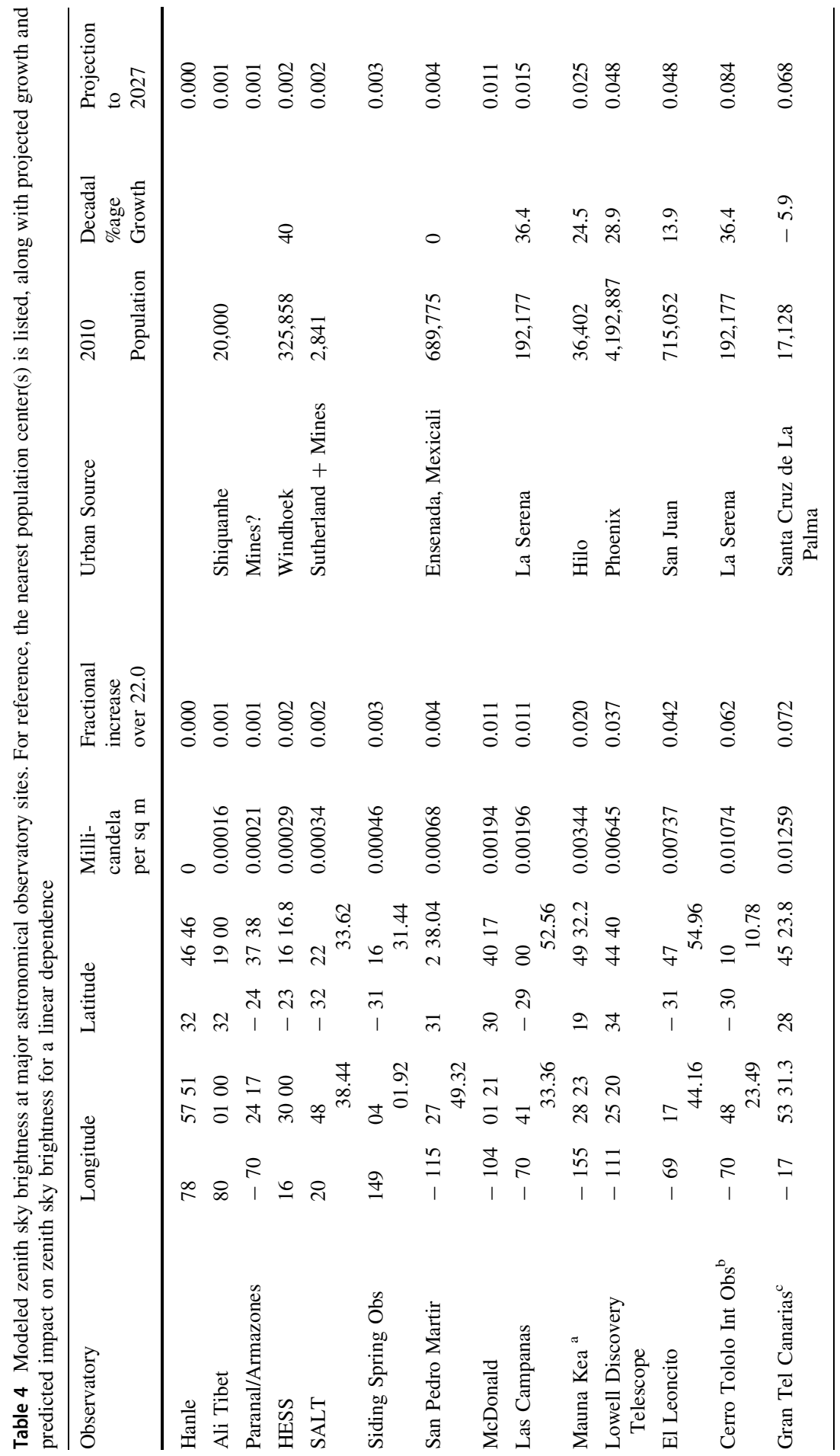




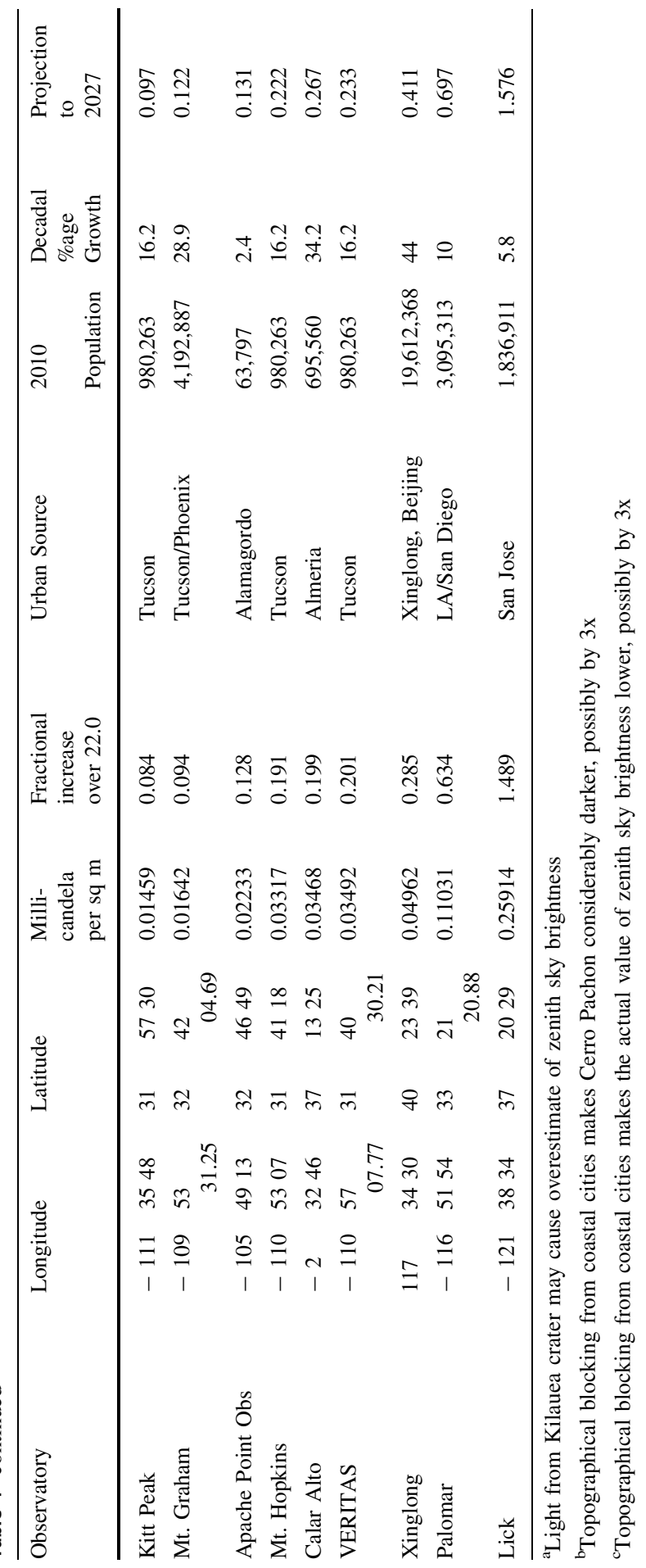


F. Falchi kindly provided the high-resolution model map data underlying the Falchi et al. (2016) analysis to RFG for extraction of the modeled values at specific locations. Table 4 shows for sites with large and moderate-aperture telescopes the artificial sky glow at zenith in milli-candela $\mathrm{m}^{-2}$ and in fractional increase over a uniform sky brightness of $V=22.0$. The list of major sites with nearly pristine conditions, i.e., with artificial zenith sky glow $\leq 1 \%$ in this particular modeling of the data includes Cerro Paranal / Cerro Armazones in Chile, Sutherland/SALT in South Africa, Ali in Tibet and Hanle in close proximity in India, San Pedro Martir in Baja California, Mexico; the HESS site at Windhoek in Namibia, and Siding Spring Observatory in Australia. McDonald Observatory in Texas and Las Campanas Observatory in Chile are right at the boundary. Note that the artificial light contribution may be overestimated for Mauna Kea Observatory, in that a major source of up-light is the caldera of the Kilauea volcano, but the lava is deep red and does not contain significant blue-green light implicit in the standard source energy distribution for the scattering model. Similarly, Cerro Pachón in Chile and La Palma in the Canaries have topological blocking that reduces the artificial zenith sky brightness considerably compared to the model.

Falchi et al. (2016) cite the criterion enunciated by the Site Protection Commission of the International Astronomical Union (Cayrel 1979):

"A contribution of 10 per cent of the natural level, which therefore worsens the basic sensitivity of a telescope by the same proportion, is generally agreed to be the maximum tolerable limit under ideal circumstances. This level of sky brightness due to artificial light is specified for $45^{\circ}$ elevation above the horizon, and for wavelengths throughout the optical spectrum from 300 to 1000 nanometers."

It is important to note that sites in proximity to light domes from large urban areas can have the contribution of artificial light at $45^{\circ}$ elevation exceed that at the zenith by 0.2-0.3 mag $\operatorname{arcsec}^{-2}$ (e.g., Walker 1973; Massey and Foltz 2000; Pedani 2009). Therefore, the threshold for considering a site beyond the IAU "tolerable limit" for deepest optical observations set by Falchi et al. (2016) of 8-10\% at zenith is reasonable, given the modest systematic effects that could lead to overestimates of zenith sky brightness increase as discussed above. Sites that fall immediately below that threshold are the Cerro Tololo Interamerican Observatory in Chile and the La Palma site with the Gran Telescopio Canarias. Working sites impacted at the threshold level are Kitt Peak and Mt. Graham in Southern Arizona. Sites more significantly impacted by urban lights include Mt. Hopkins in Southern Arizona, Apache Point Observatory in Southern New Mexico, Calar Alto in Southern Spain, and Xinglong Station with the LAMOST Observatory outside of Beijing. Historic sites with extremely strong glare from artificial light under clear conditions are Palomar and Lick Observatories in California.

In Garstang's (1989b) projections for sky brightness increases at Observatory sites, he assumed that artificial sky glow from an urban area depended linearly on population. That assumption will not be strictly true if regulations change the fraction of up-light because of better regulated shielding or if there are other changes in lighting technologies. The latter case is graphically illustrated by Falchi et al. (2016) in their Fig. 5, if the blue light contribution of LEDs replaces the much lower contamination from high pressure sodium. To assess the impact of population 
growth on major observatories, the population of the nearest large urban center(s) is listed in Table 4, along with the percentage decadal population growth. On the highly simplified model that the strongest current source (city) will dominate the artificial light, that the decadal growth will continue for another decade at the same rate, and that the combined effects of LED conversion and better shielding are negligible, the final column lists the projected percentage addition of artificial light at zenith for the same sites ten years from now. Not surprisingly, only the most nearly pristine sites stay in that condition, while those currently impacted by urban areas show a relative increase of $20-30 \%$, driven by annual population growth and increased per capita outdoor lighting usage. Any policy goal of holding the artificial light contribution at constant or decreasing levels must counteract the impact of both population increase and the lower cost per lumen of solid-state lighting driving over-illumination. The ongoing monitoring by downward-looking satellites can provide important observational checks on both those trends. Bará et al. (2020b) derived fast Fourier transform techniques that greatly simplify the computational aspect of the required modeling.

\section{Public policy, codes, and enforcement}

\subsection{Light pollution/lighting regulation}

Population and economic growth have been accompanied by an increase in outdoor lighting in both urban areas and smaller communities in proximity to major observatories. Observatory management realized that community engagement and lighting regulation would be required to preserve dark sky access. The earliest example is the ordinance from the City of Flagstaff in 1958 prohibiting the use of searchlights (Lockwood 2002). Kitt Peak, Steward, and Smithsonian Observatories undertook a campaign of community engagement with the City of Tucson and surrounding Pima County to develop outdoor lighting ordinances in the early 1970s, while the Kitt Peak National Observatory 4-m and other telescopes were under construction (e.g. Walker 1973).

Today, light pollution control and the specific protection of major observatory sites are enshrined in local and national regulations. The current status is summarized in Table 5. The general approach in the United States is to make these regulations part of the local Zoning Code, which regulates the usage of property. The majority of other codes are established for protection of a specific astronomical site and/or are part of environmental or sustainability regulation.

There are many common elements to these regulations. Almost all are prospective only, applying to new development or major redevelopment of properties. An exception is the Andalucia statute for protection of Calar Alto, which did require that all fixtures with greater than $25 \%$ up-light be removed within three years of enactment in 2010. Most require fully shielded fixturing, those that allow no light to be directly radiated above horizontal. As discussed above, radiation into directions immediately above horizontal has the greatest impact on observatory sites outside of the urban area from which it emanates (Luginbuhl et al. 2009a). The 


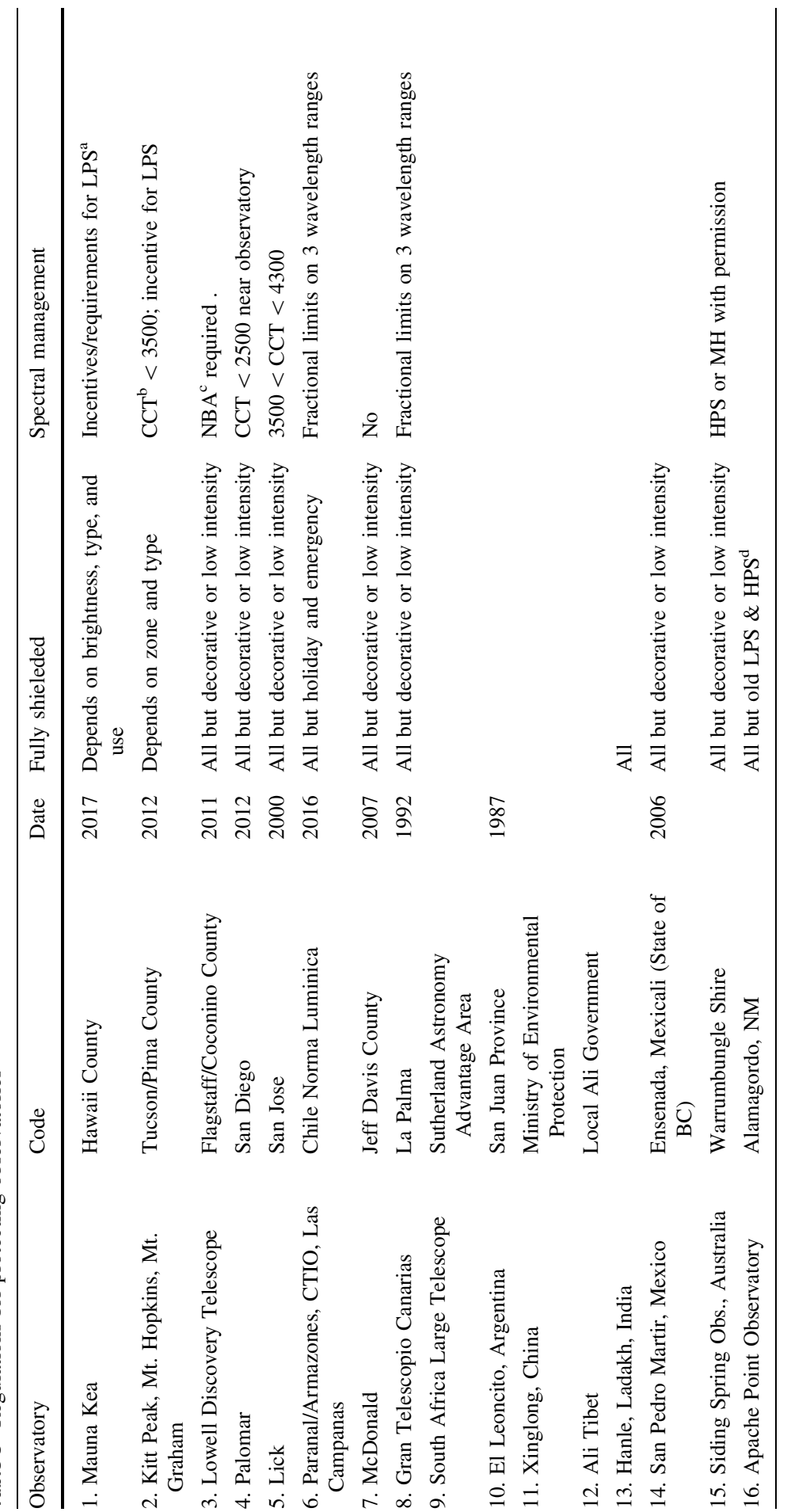




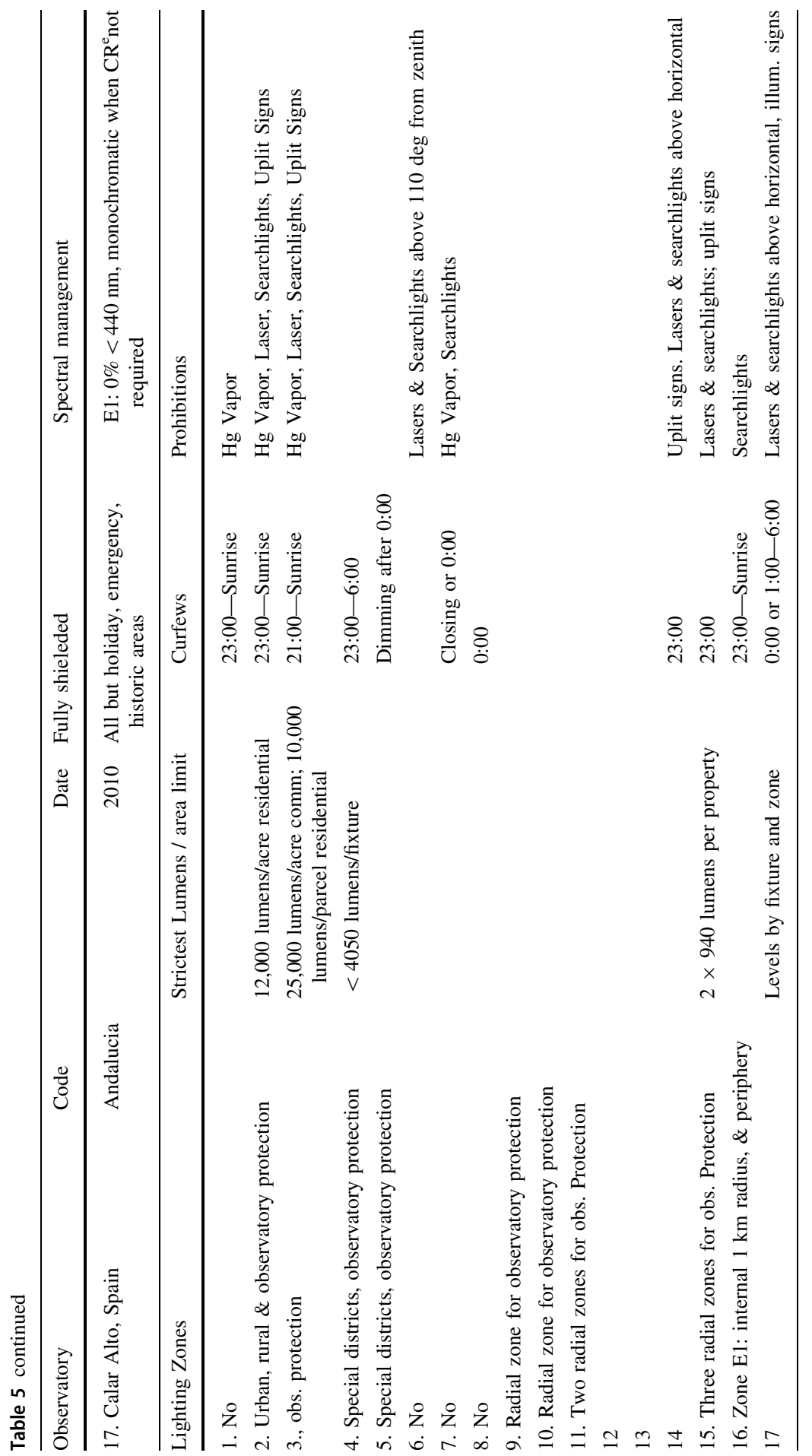




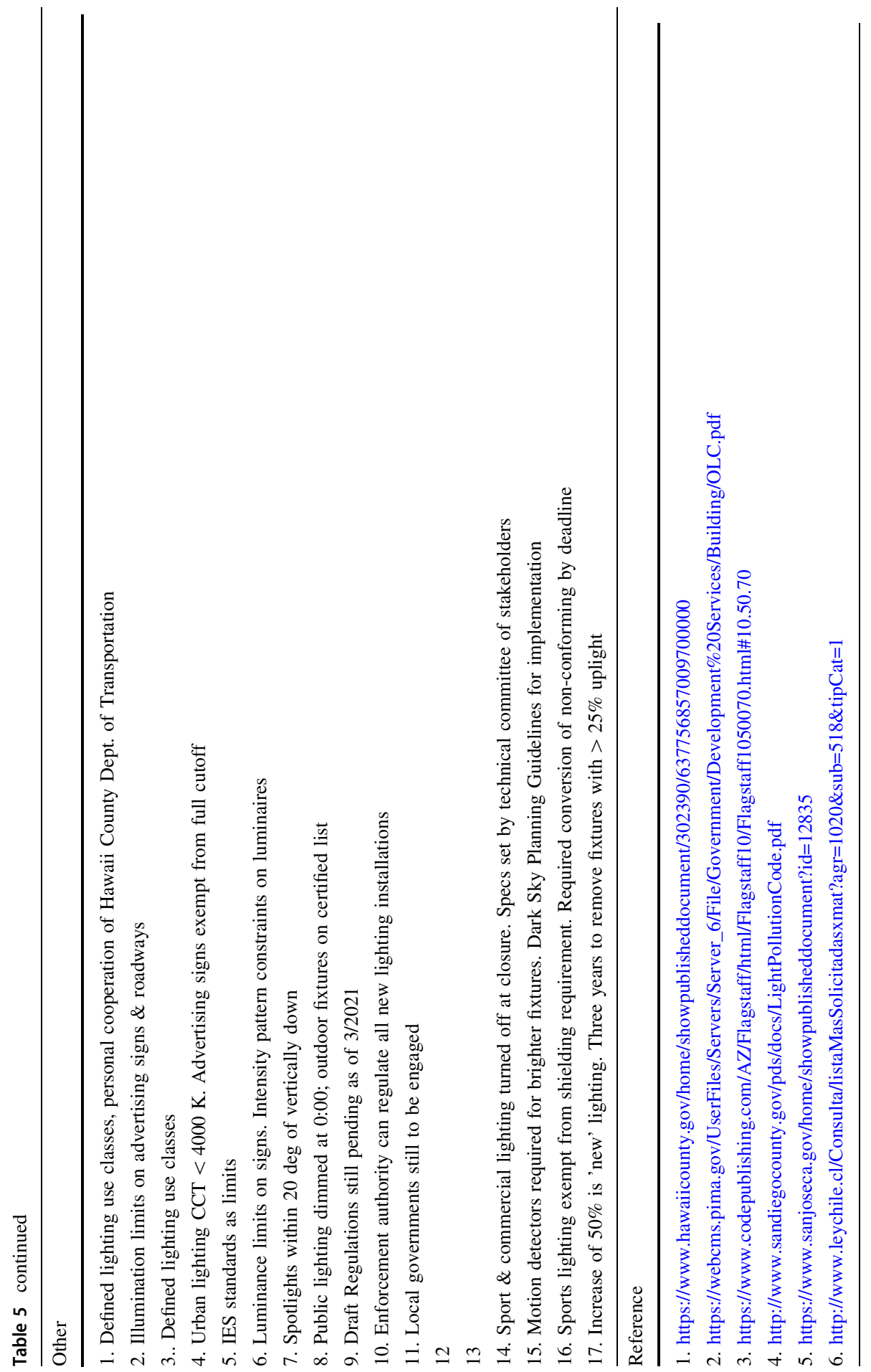




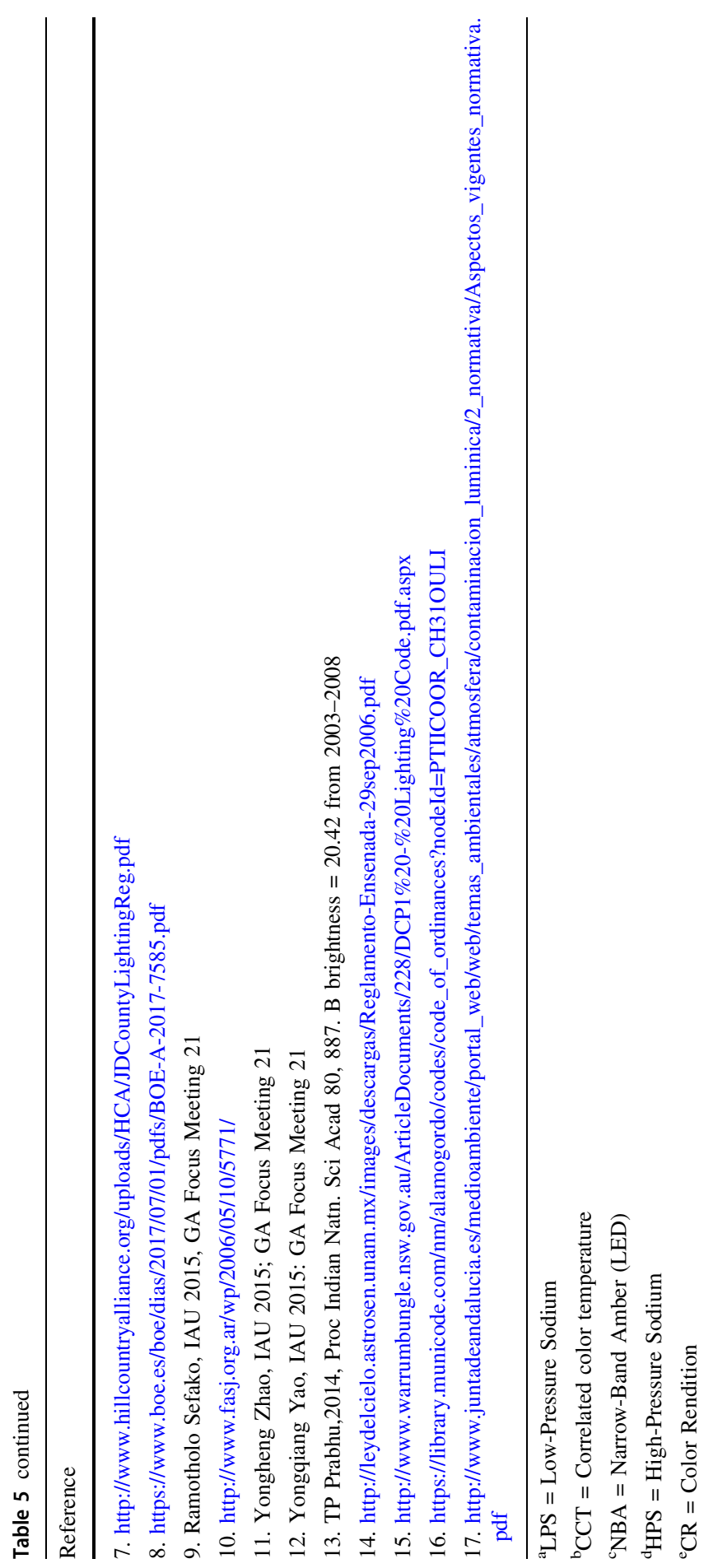


IAU Commission on Site Protection made full cutoff its highest priority for protection of dark skies (Green 2012). Exceptions are granted for low-intensity and decorative fixtures. Searchlights and bottom-lit signs tend to be specifically prohibited.

Given the $\sim 1 / r^{2}$ impact of light sources, many codes create special zones of protection immediately around observatory sites. Many restrict the lumens per fixture depending on the radial zone around the observatory. In Arizona, Flagstaff, Coconino County, Pima County and City of Tucson codes restrict the total number of lumens per acre (hectare) for new development, depending on distance to major observatories and land use (commercial vs. residential). Many codes offer incentives to deploy fully shielded fixtures by increasing the allowed limit on lumens if the installation is $100 \%$ full cutoff. All grant exceptions to the full cutoff requirement for emergency installations, and many exempt roadway lighting.

As discussed above, with the new generation of solid-state LED lamps, there is a growing issue of spectral management, which typically requires an update of existing codes. The Chilean national code (the Norma Lumínica) specifies the fraction of total energy output allowed in three specific wavelength ranges:

300-379 nm: luminous intensity $<0.15 \times$ luminous intensity for $380-780 \mathrm{~nm}$.

$380-499 \mathrm{~nm}$ : luminous intensity $<0.15 \times$ luminous intensity for 380-780 nm.

780-1000 nm: luminous intensity $<0.5 \times$ luminous intensity for 380-780 nm.

The new proposed Norma Lumínica sets much tighter limits on the fraction of the radiation outside of the 500-780 nm band (MMA 2021).

The code for protection of Calar Alto in southern Spain allows 0\% of the energy below $440 \mathrm{~nm}$, and mandates a monochromatic (or narrow-band) source when color rendition is not critical to the use. Others create incentives by allowing more lumens of low-pressure sodium than for other spectral sources. U.S. codes have started placing limits on the correlated color temperature rating of lamps (typically applied to LED), from San Diego County requiring $<2500 \mathrm{~K}$ near the observatory, and the Southern Arizona codes setting CCT $<3500 \mathrm{~K}$, while the City of Phoenix just purchased roadway lighting with $\mathrm{CCT}=2700 \mathrm{~K}$.

Time management of artificial illumination is also critical for astronomical site protection. Most codes contain a curfew, typically 23:00 or midnight, at which commercial illumination must be turned off or greatly reduced in intensity. La Palma also requires that lighting of public spaces be dimmed after midnight. Warrumbungle Shire took a contemporary approach to protection of Siding Spring Observatory by requiring that brighter fixtures be activated only by motion detectors.

The following sections provide examples of locations that have had some measure of success in successful light pollution mitigation. There is one location (Flagstaff, Arizona) where all three aspects of the problem-technical standards; identification of a threshold; successful implementation-have to a degree been successfully addressed, with measurable results. The degree of success for most is uncertain, due to the lack of ongoing measurement or monitoring as noted previously. Nonetheless, they may serve as illustrative examples of aspects of effective regulatory approaches and overall strategies. 


\subsection{Two detailed examples}

\subsubsection{Flagstaff, Arizona USA}

In 1989 Flagstaff adopted the first known outdoor lighting code with standards based on limiting sky brightness at an observatory site. This code was matched by a nearly identical code in the surrounding Coconino County adopted in the same year. These remain to our knowledge the only lighting codes with standards designed to achieve a sky brightness management target. Besides the innovative limits on overall lumen amounts (see below), these codes also included strict standards for the use of low-pressure sodium for "Class 2" lighting (defined as "lighting for general illumination" such as for parking areas, security, and roadways), and strict shielding standards.

The lumen limits were devised to limit the increase in total zenith sky brightness over the U.S. Naval Observatory Flagstaff Station (NOFS) to 30\% over the then current condition, which meant, based on then current sky brightness measurement and sky brightness modeling, an increase from $V \sim 21.65$ to $V \sim 21.37 \mathrm{mag} /$ $\operatorname{arcsec}^{2}$. Modeling of the expected sky brightness increase from development of vacant commercial/industrial properties, performed by R. Garstang using an early version of his model (Garstang 1986) were used to establish a limit of 25,000 (lamp) lumens per acre $(10,100 \mathrm{~lm}$ per hectare) within a zone of 2.5 mile radius centered at NOFS. Combined with the available area for development within this zone, this lumen per acre translated to approximately 5,000,000 $\mathrm{lm}$.

The lumen caps in further zones were increased in steps by factors of two, to 50,000 and 100,000 lumens per acre, as sky brightening impacts of development in these outer areas were decreased by distance. It was anticipated in a rough sense that lighting added by additional development of vacant land in these outer zones would be offset by redevelopment and replacement of old lighting under the tighter standards of the new code.

It is important to note that the conceptual basis for the lumen cap in the innermost lighting zone was not lighting needs or lighting industry recommendations. It was understood that the limit would have implicit impacts on possible land uses or typical development practices. In the more distant zones, with limits of 50,000 $\mathrm{lm}$ per acre and above, arguments can be made that industry-recommended illumination needs for most uses can be met with minimal adaptation (IDA 2002), and experience since the adoption has borne this out. This is one instance showing the result of a policy balancing the competing purposes of development and dark sky protection.

The lighting code has been successful in achieving its goals of limiting sky brightness increase at NOFS. Measurements made in June 2015 indicate an actual decrease of approximately $10 \%$ compared to the measures made in the late 1980s. This decrease is thought to arise from the further shift of Flagstaff outdoor lighting based on the 1989 lighting code standards and most roadway lighting to lowpressure sodium, which has decreased impact in the Johnson V band, the beneficial effects of improved shielding, not fully understood in the modeling effort in the late 
1980s, and the effects of near-ground blocking, not understood or included in the 1980s modeling.

Following the June 2015 sky brightness measures, and using improved light pollution modeling, NOFS has recommended, and the community planning bodies have supported, the revision of the Flagstaff and Coconino County lighting codes to achieve a new management target of only a $10 \%$ increase over current sky brightness conditions. These amendments involve conversion of the former lamp lumen standards to fixture lumens (amounting to a $0.7 \times$ decrease); stricter shielding requirements in the closest Lighting Zone; reduction of the lumen limit in the outermost zone (formerly 100,000 lamp lumens or 70,000 fixture lumens per acre) to the values of the next innermost zone (35,000 fixture lumens per acre); and the elimination of the requirement for roadway lighting in residential areas. These amendments are in process as of spring 2021.

This result demonstrates not only the effectiveness of the technical aspects of the lighting code standards, but also the successful implementation and maintenance of the standards through the governing process. The most critical aspect of this successful implementation is the ongoing engagement of observatory staff at both NOFS and Lowell observatories with the local planning process, as well as the efficacy of a citizen-based group advocating for dark skies based on community values (and not just economic benefits of having professional astronomical observatories). This engagement involves attendance at hearings and both public and where possible internal planning staff meetings where lighting plans or rezoning are considered, consultation with planning staff to assure understanding of and effective implementation of the codes, and assistance in developing code amendments where misunderstandings develop or technology changes necessitate. This engagement is a crucial aspect to successful light pollution mitigation, one rarely achieved.

\subsubsection{Maunakea, Hawaii USA}

6.2.2.1 Approaches to local regulation Maunakea has been protected by a strong lighting ordinance since 1990. The ordinance has been recently adapted to allow use of LEDs. The core component of the original lighting ordinance was widespread use of low-pressure sodium (LPS) lighting for all applications where color rendition was not important. LPS lighting has several important advantages for astronomy. The first is that it is nearly monochromatic, with nearly all of the energy emitted near $589 \mathrm{~nm}$ wavelength (amber color). This means that for spectroscopic observations with a telescope, only one wavelength is affected, and that same wavelength is already compromised naturally by sodium emission in the upper atmosphere from sodium deposited by micrometeorites. The second reason relates to Rayleigh scattering. At most major observatory sites (particularly the island sites-Hawaii and La Palma), the air is clean with very little aerosol. The dominant scattering mechanism for artificial light is therefore Rayleigh scattering by air molecules, which is very strongly wavelength dependent. Blue light at $450 \mathrm{~nm}$ scatters three times as much as LPS light. LPS light is nevertheless close in wavelength to the photopic peak in the eye's sensitivity at $555 \mathrm{~nm}$, so it is effective for human vision. 
The disadvantage of LPS lighting is that it is difficult to control. Early versions of the lighting ordinance on the Island of Hawaii did not require full shielding of lights because of the limited availability and efficiency of fully shielded LPS fixtures. Later updates to the lighting ordinance did require full shielding of all lights. Luginbuhl et al. (2009a, b, c) showed that full shielding of light sources is one of the most important tools for protecting the night sky. Light directed at small angles above the horizontal is particularly damaging. The lighting ordinance on the Island of Hawaii now requires that all artificial light sources are fully shielded, and emit no light above the horizontal plane.

Another important aspect is the amount of light used (cf. Sect. 3.2). On the Island Hawaii, streetlights are commonly mounted on utility poles, which have spacing that makes it difficult to achieve uniformity and lighting levels that are recommended by lighting organizations such as CIE and the Illumination Engineering Society of North America (IESNA). The lighting is nevertheless adequate and safe. Electricity is very expensive on the Island of Hawaii, with prices sometimes as high as US\$ $0.50 / \mathrm{kWh}$. This provides strong additional motivation for energy efficiency (one of the arguments supporting the use of LPS), and to use only the amount of light needed, and to not over-light.

Dimming of light sources is another important tool to protect the dark night sky. Lighting levels are often set by counting the number of pedestrians in the early evening. There are many fewer pedestrians (and cars) later at night, so following the pedestrian count rationale, lights can be dimmed. Dimming is expected to be implemented on some state highways, and in other counties in the state of Hawaii. Dimming of LEDs is relatively simple; it is much more difficult to dim arc discharge lamps such as LPS and high-pressure sodium (HPS). On the Island of Hawaii, street lighting levels on county roadways are already close to minimum levels recommended by the lighting industry, and it is unlikely that these lights can be further dimmed. Haleakala observatory on the Island of Maui is a smaller observatory that has been protected by a much weaker lighting ordinance. The core requirement of the Maui County lighting ordinance is for full shielding of lights. However, metal halide lamps, which are very damaging, are not required to be shielded (because of their use at recreational facilities, and the cost associated with replacing them with shielded fixtures). Maui's lighting ordinance has very weak spectral power distribution limitations. It urgently needs to be updated, particularly to adapt to the use of LED lighting.

Two techniques are possible. The blue LED light (from a white LED) can be filtered out. Or LEDs that intrinsically emit little or no blue light can be used (e.g., amber LEDs). Due to energy efficiency considerations, the approach that has been adopted on the Island of Hawaii for streetlights is use of filtered LEDs. A filter is used that absorbs essentially all light shortward of $500 \mathrm{~nm}$. The resulting light has a greenish-yellow hue if a $5000 \mathrm{~K}$ white LED is used as the base illumination, or a yellow hue if a $3000 \mathrm{~K} \mathrm{LED}$ is used as base illumination (the spectra of these filtered LEDs are shown in Fig. 4 as FLEDcw and FLEDww, respectively). The similarity of the color of amber LED lighting and the amber (yellow) traffic signal was raised as a concern on the Island of Hawaii. This results from many years of use of partially shielded LPS lights allowed under the early code standards. The color of 
an LPS light is indistinguishable from that of an amber traffic signal. Likewise, the color of an amber LED is indistinguishable from the amber traffic signal, because the amber LEDs are used in traffic signals. Fortunately, full shielding of streetlights reduces the likelihood that a streetlight could be mistaken for a traffic signal. Phosphor converted amber LED and filtered white LEDs both have color rendering indices of approximately 60. Both sources are deficient in blue light. Filtered LEDs are now being used for street lighting on the Island of Hawaii.

In the United States, county and state laws do not affect federal installations. County laws for the County of Maui do not affect lighting on the Island of Oahu (where Honolulu is located); Oahu makes the night sky bright in the northwest seen from Haleakala. State facilities, such as airports and harbors, may try to exempt themselves from County lighting requirements. And federal facilities, such as the Pohakuloa Training Area, are not required to follow the County lighting ordinance.

Photographs of Hawaii obtained by astronauts on the International Space Station have shown that the airports and harbors are among the brightest source of light on the Island of Hawaii, and careful lighting there is vitally important. The army training area at Pohakuloa is located only $10 \mathrm{~km}$ from the observatory. Because of its close proximity, it is critically important that their lighting is state-of-the-art in terms of reducing impact to the observatory.

In Hawaii, state laws have been established that require the state Department of Transportation to follow county lighting ordinances for highway, airport and harbor lighting. State coastline lighting laws have also been enacted. A state night sky protection advisory committee has been established, and a state law requiring all state lighting to be fully shielded and to have correlated color temperature $\leq 4000 \mathrm{~K}$ is now in place. The state law will begin to address the impact that the bright lights from Honolulu have on Haleakala observatory and on the more distant Maunakea observatory. Although the Pohakuloa Training Area is not required to follow the county lighting ordinance, they are voluntarily complying. The army minimizes impact on the night sky by using careful shielding and selecting light sources that minimize emission at blue and green wavelengths.

Hawaii has numerous endangered birds and turtles, and many of these animals are profoundly affected by light at night. The endangered Newell's Shearwater, found mostly on the Island of Kauai, circles around an unshielded light until it is exhausted, at which time it collapses to the ground near the light, where it is vulnerable to predators. The US endangered species act produces strong motivation to use proper shielding of lights. As a result, almost all lighting on the Island of Kauai is fully shielded, despite there being no observatories on that island. The lighting requirements for protecting endangered species and for protecting the observatories are closely aligned, both in terms of shielding and spectral energy distribution. Endangered turtles are much more affected by white light than by amber light.

It is important to update lighting ordinances regularly to adapt them to changes in lighting technology and other changes. The lighting ordinance on the Island of Hawaii is presently being revised. One change that has become necessary is to limit nighttime light emission from greenhouses. Grow lights are sometimes used in greenhouses at night to increase crop production. These can have a strong impact on 


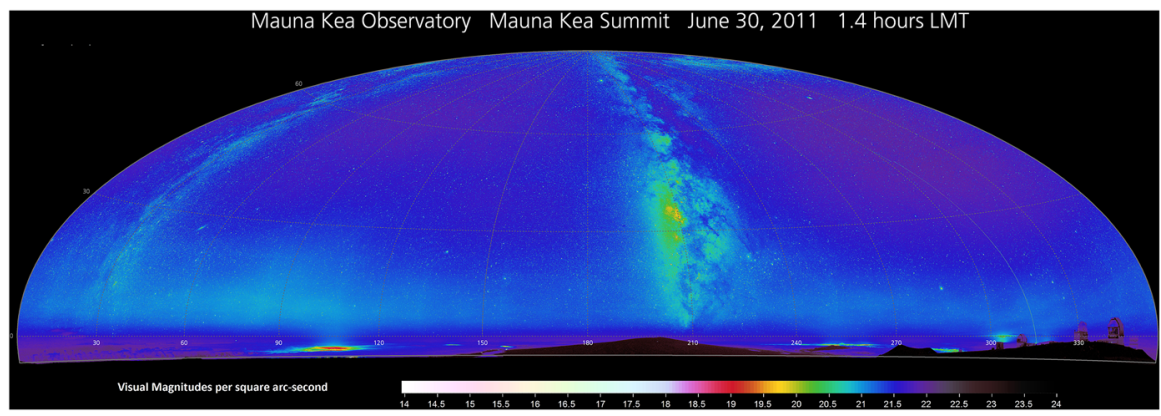

Fig. 12 The night sky seen from Maunakea, as measured by the night sky team of the U.S. National Park Service. It shows a night sky with very little contamination from artificial light sources. The sky is darker than at any National Park in the United States, including Denali National Park in Alaska. Denali National Park has essentially no artificial light, but the sky is brighter there because it is closer to Earth's magnetic pole, so airglow is stronger. Honolulu is located approximately $300 \mathrm{~km}$ from Maunakea. The impact of Honolulu's lighting on the night sky over Maunakea is small

the night sky, and so should be appropriately shuttered at night to prevent escape of light. Architectural lighting is often not properly shielded, and is often damaging to the dark night sky. The revised lighting ordinance will explicitly prohibit illumination of rooftops.

6.2.2.2 Results in night sky protection The Island of Hawaii has a population of nearly 200,000. As a result of the strong lighting ordinance on the Island of Hawaii, the night sky over Maunakea observatory remains very dark, and is well suited to the demanding requirements of the deep sky astronomy performed by the large telescopes located there (Fig. 12). In fact, the night sky over Maunakea is among the darkest night skies in the world. Astronomical observatories benefit from nearby communities, because these communities provide infrastructure such as schools, shops, and medical facilities for the staff that work at the observatories, as well as providing other logistical benefits such as transportation. However, very careful and strong lighting regulations are required for observatories to successfully coexist with urban development. In contrast, the Island of Maui has a much weaker lighting ordinance, and as a consequence, the night sky over Haleakala observatory is less dark (Fig. 13) Haleakala is also closer to the city of Honolulu and the Island of Oahu (population approximately 1 million), where the lighting is less well regulated. At a distance of approximately $180 \mathrm{~km}$, Honolulu and Oahu have a significant impact on Haleakala's night sky, and make the northwestern sky brighter.

Flagstaff provides a second example at a moderately polluted site, and provides a quantitative insight into what is achievable through effective sky glow mitigation. Measurements also made by the U.S. NPS (see Fig. 14) from $27 \mathrm{~km}$ outside of Flagstaff (population 70,320) show that its integrated $\mathrm{V}$ band artificial sky brightness is less than $1 / 10(1 / 11.9)$ the integrated sky brightness over Cheyenne Wyoming (population 63,335) as observed from $31 \mathrm{~km}$ (Pipkin et al. 2017). Though this is a single point comparison, the long history of aggressive light pollution 


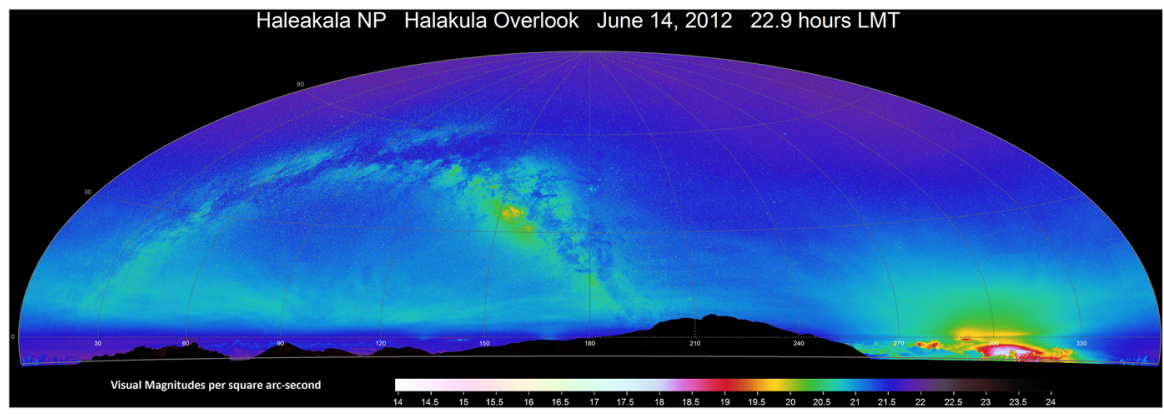

Fig. 13 The night sky seen from Haleakala. The sky is brighter than seen from Maunakea. Maui is a smaller island and its population lives closer to the observatory. The bright sky in the northwest is caused by light from the Island of Oahu (where there are no strong lighting regulations)

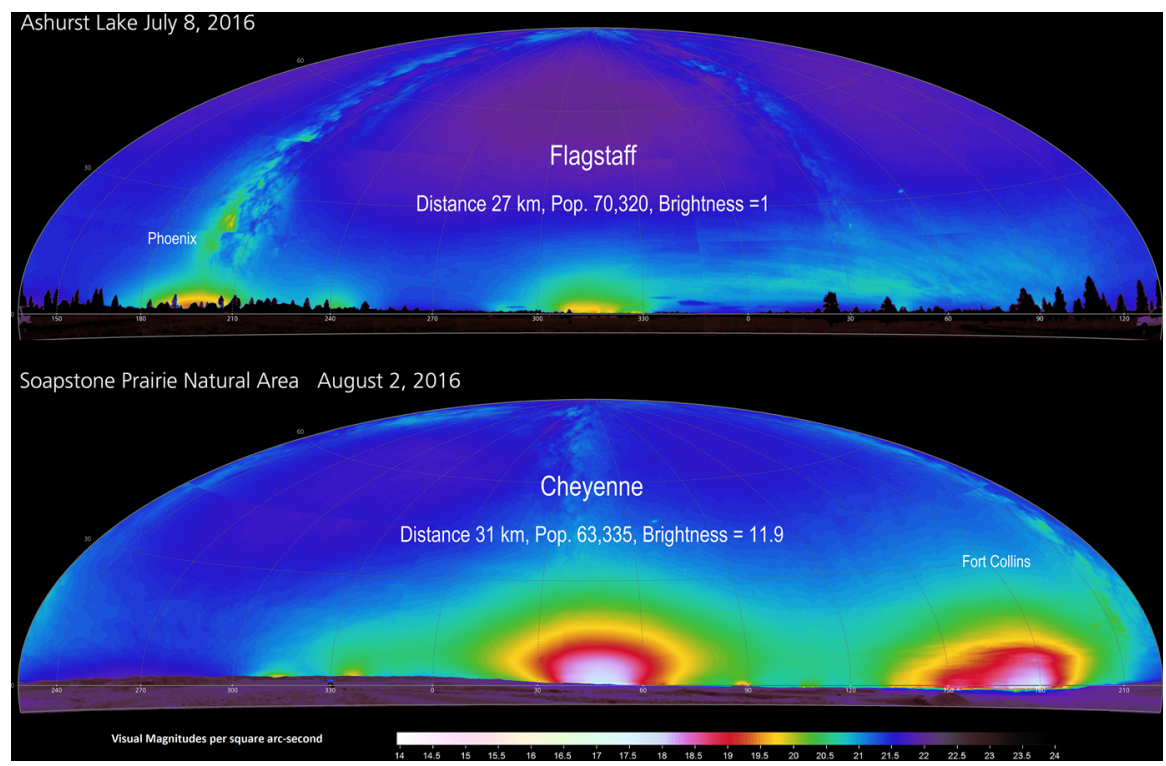

Fig. 14 U.S. NPS all-sky image comparison of images taken $27 \mathrm{~km}$ outside of Flagstaff, AZ (upper) and $31 \mathrm{~km}$ outside of Cheyenne, WY. The two cities have similar populations, but Flagstaff has a history of aggressive light pollution control efforts

control efforts in Flagstaff contrasted to a similarly-sized community with no particular historical concern for light pollution shows that dramatic reduction may be more widely achievable. 


\section{Satellite constellations in low-Earth orbit}

With the launch of the first tranche of sixty SpaceX Starlink telecommunications satellites on May 23, 2019, the astronomy community was suddenly awakened to the reality and potential of mega-constellations in low-Earth orbit (LEO) (Wall 2019). The impact on observations, particularly those subtending wide angles, arises from the brightness of the reflected sunlight and the frequency of satellite passage through any targeted field of view, should all proposed constellations be fully built out.

The Starlink satellites were of naked-eye brightness in their post-launch configuration prior to their boost to final orbit, and tightly clumped, providing wide visibility to sky watchers (Hall 2019). On orbit, they were measured to be in the 4th to 6th visible magnitude range, depending on phase angle (Walker et al. 2020b). Lab simulation of trails of equivalent brightness on the CCD detectors to be used by the Vera Rubin Observatory produced cross-talk ghost trails that could not be consistently modeled and removed. The required masking would have eliminated substantial area parallel to each recorded satellite trail, compromising the ability to pursue key classes of objects in twilight, such as near-Earth asteroids and rapidly fading transients (Tyson et al. 2020).

Public expressions of concern by the astronomy community and the willingness of SpaceX management to reduce the impact of the Starlink constellation on observatories led to direct interaction. The result was the establishment of an approximate benchmark for reflected sunlight, below which the crosstalk from satellite trails on the Vera Rubin Observatory CCD detectors could be calibrated and removed. That target was $V \sim 7$ th mag, corresponding to $44 \mathrm{nW} / \mathrm{sr}$. (The dependences on distance, angular speed and image concentration make that relation scale as $1 / r_{\text {orbit }}$ (Walker et al. 2020b). To meet that goal, SpaceX experimented with both a low-reflectivity coating (DarkSat) and a sun shield (VisorSat). The measured results showed promise (Tregloan-Reed et al. 2020). In addition, the brightness of the immediate post-launch phase can be reduced by rolling the spacecraft to present a smaller cross-section for reflection before orbital raise (Walker et al. 2020b).

The outlook remains concerning, should all the planned constellations of LEO telecommunications satellites come to fruition. A sampling of currently registered plans is found in Table 6; the information changes on the timescale of weeks. They represent most of the 60,000 + LEOSats proposed to be launched before 2030 . Several groups are now performing simulations to assess the impact of such constellations (Hainaut and Williams 2020, McDowell 2020, Ragazzoni 2020, see also Walker et al. 2020a) The general conclusion is that constellations in low orbit ( $\sim 600 \mathrm{~km}$ or lower) have their impacts largely in twilight, where some $5 \%$ of the constellation is above the horizon, but mostly near the horizon (50\% below $20 \mathrm{deg}$ elevation). Because of the conical shape of the Earth's shadow, satellites in higher orbits, such as those of OneWeb, are visible longer; in summer some can be in view all night. A simulated example shows that there would be two satellite trails per minute per square degree at the position of the Large Magellanic Cloud in summer (Walker et al. 2020a). For many directions and latitudes, that frequency of trails 
obtains when the Sun is near 18 deg below the horizon (Walker et al. 2020b). Also concerning is the alternate consideration of an integrated ensemble effect for reflected and scattered sunlight from the full distribution of sizes of objects in LEO. If their streaks subtend a small fraction of a detector's angular resolution element, they may already be contributing a $10 \%$ increase of diffuse zenith luminance over the natural baseline (Kocifaj et al. 2021).

Ground-based astronomical observing is currently at a watershed. Night-time images without the passage of a sun-illuminated satellite will no longer be the expectation. At the time of this writing, two conferences have been held to explore the scientific consequences, the possibility of mitigation, and the approach toward policy. SATCON 1 (Walker et al. 2020a) focused on technical mitigations for satellite operators and the operational and data processing modifications required for astronomers. A workshop on Dark \& Quiet Skies was sponsored by the United Nations Office of Outer Space Affairs, the International Astronomical Union and the Astrophysical Institute of the Canaries. It was aimed at developing options to present to the UN Committee on the Peaceful Uses of Outer Space for an international approach to mitigation. The most effective approach is to limit orbital height and the number of constellation elements, as well as limiting reflectivity to stay well below the threshold for the Vera Rubin Observatory detectors, and by extension, avoiding saturation for most other telescopes. Improving predicted positions for LEOSats by about an order of magnitude in the cross-track direction can be a collaborative effort with industry and the astronomical community, and is required to provide useful information for dynamic scheduling when possible.

Table 6 Planned LEO constellations

\begin{tabular}{llll}
\hline Constellation & Operator & Number & Orbital heights (km) \\
\hline Starlink2 & SpaceX & 30,000 & $328-614$ \\
Kuiper & Amazon & 3,236 & $590,610,630$ \\
WorldVu & OneWeb & 6,372 & 1200 \\
Telesat & Telesat (Canada) & 1,671 & 1325 \\
Kepler & Kepler & 360 & 600 \\
Viasat & Visasat & 288 & 1300 \\
Hongyun & CASIC & 864 & 1165 \\
Hongyan & CASC & 320 & 1100 \\
Galaxy Space & GalaxySpace China & 650 & $630 ?$ \\
GW-A59 & GW-China & 6,080 & $508,590,600$ \\
GW-2 & GW-China & 6,912 & 1145 \\
V-band constellation & Boeing & 147 & 1056 \\
SpaceNet & Astrome India & 600 & 1400 \\
SpaceMobile & AST\&Science & 243 & 735 \\
Hanwha & Hanwha (Korea) & 2,000 & - \\
Lynk & Lynk & $1000 \mathrm{~s}$ & 500 \\
\hline
\end{tabular}


Development of community-accessible user software for identifying and masking satellite trails was deemed meritorious of support. Deeper investigation will be required to understand whether losses of scientific information extend beyond the linear area impacted directly by the bright trail of reflected sunlight. Examples of concern include shape analysis of faint galaxies for dark matter lensing or time series monitoring of crowded star fields.

The challenge to the astronomical community is similar to that for protection of the dark night sky from ground-based sources of light pollution. There must be a broad recognition of the need to balance commercial interests with scientific concerns for use of the "commons" of low-Earth orbit.

\section{Conclusions and options for astronomy}

The quantitative understanding of the influence of lighting amounts and characteristics on sky brightness, summarized in Sect. 4, provides critical quantitative guidance in the development of the technical aspects of lighting regulations. But successful mitigation of light pollution is unfortunately more complex than luminous flux, scattering, and atmospheric optics. Without the identification of artificial sky brightness or light pollution as a genuine problem requiring a solution by regulatory bodies, technical solutions will not be legally implemented, or even if implemented too seldom supported with the resources necessary to make them effective.

In the process of building consensus that light pollution is a genuine problem, critical deficiencies have been the limited nature of sky brightness measurements and lack of clearly articulated sky brightness thresholds or limits. Not only do these deficiencies mean that mitigation strategies are difficult to develop based on quantitative understanding of the current or desired condition, but the fact that the data are not being processed, calibrated and reported on a regular basis can be interpreted to mean that the proponents do not regard the problem as critical. Even professional observatories rarely calibrate and publish such measurements. Though the recent work of groups raising awareness of light pollution such as the International Dark-Sky Association, and most particularly resources like the New World Atlas of Artificial Night Sky Brightness (Falchi et al. 2016) are beginning to raise more general awareness of the dimensions of the problem, and identify qualitative thresholds (e.g. Milky Way visibility), much more is needed, particularly at the local level where light pollution mitigation regulations are implemented.

The Dark and Quiet Skies Workshop set the ambitious goal of slowing, stopping, and reversing the growth of ALAN in the skies above professional observatories (as well as environmentally critical areas) within a decade. The proposal is to accomplish that goal with two new approaches to policy and regulation. The first is to create regional lighting control plans unique to each observatory, based on radiative transfer modeling specific to the sources of light from developed areas and the topography of the observatory region. The second is to define all usage zones requiring lighting at night dynamically, based on traffic and time of night. The latter is consistent with the new LED control technologies, and is likely to have a 
pronounced impact on artificial sky brightness, particularly as human activity sharply reduces later in the night.

The approach to establishing appropriate management strategies from the astronomical perspective is based on a sky brightness threshold or limit determined by observatory observational needs, with lumen limits (and other lighting standards) and locations determined such that long-term lighting changes meet the goals of gradual reduction of ALAN. To date, with rare exceptions, such thresholds have not been identified. Thus lighting amounts are often unaddressed, or if addressed follow an approach based on recommendations of the lighting industry. Since this approach is based on use-specific limits and not global effects, though it may arguably reduce sky brightness increase, it does not effectively limit sky brightness increase.

The revolution in solid-state LED lighting offers both promise and peril for the future of observations requiring the darkest skies at major observatories. With modern fixture design and recognition of research in human visual response, outdoor spaces can be lit with fewer total lumens. The absence of a warm-up requirement, the possibility of dimming, and mixture of narrow and broad-band SPDs create options for automatic control to cover a range of traffic and safety requirements while minimizing full-spectrum intensity when not needed.

The rapid conversion from the dominant high-pressure sodium to LEDs introduces continuum light at blue and green wavelengths that was not formerly present, subject to greater scattering (at short distances) and posing the threat of contamination to formerly untouched regions of the optical spectrum. With greater luminous efficiency, there is the temptation for government officials to choose brighter illumination than the fixtures being replaced, because they can still save on power consumption.

The Commission on Site Protection of the International Astronomical Union prioritizes three short-term mitigation measures (Green 2012):

1. Fully shielding fixtures for no light propagated above horizontal.

2. Spectral management, with the narrowest possible bandpass for lighting closest to observatories and wider-band sources used only when the case is made for the need for color rendition and only with the lowest commercially viable CCT.

3. Appropriate usage: curfews, areal zones around observatories with lumens caps, employment of modern technology to enable motion-activation, reversion to monochromatic when color rendition not needed, etc.

The Dark \& Quiet Skies workshop (Walker et al. 2020b) produced a more comprehensive recommendation for consideration by the United Nations. The proposal is that the rate of increase of artificial skyglow at observatories be slowed, stopped, and reversed within a decade. Achieving that goal requires a regional lighting plan based on radiative transfer modeling for the sources and topography unique to each site. A key aspect is dynamic definition of usage zones, dependent on traffic and activity. Active lighting controls then adjust the levels to the minimum required for safety as the night goes on. A near zone around each observatory is strongly protected with minimal outdoor lighting allowances. The IAU 
recommendations above then form the basis for the actual lighting implementation in the surrounding region.

Competing economic interests and varying attitudes toward the impacts of regulation make the arguments for dark sky protection around major observatories less than self-evident to many policy makers. Professional astronomers therefore, cannot assume that site protection is solely the responsibility of observatory management. For the special needs for extremely low lighting levels and monochromatic sources to be maintained and enhanced in regulation, strong public support is required. Many valuable sites risk being compromised over time by the growth of population or commercial (mining) activity without public action to reverse the trend of brightening artificial sky glow.

Astronomers must provide quantitative thresholds, convincing calibrated data, and follow-through monitoring to create the basis for meaningful regional lighting plans that actually lead to long-term, recorded reductions in artificial sky brightness. The maintenance of our unique optical windows to the Universe against that trend of increasing night sky contamination depends on broadly educating the public. That task falls on the entire astronomical community and requires a sustained and coherent message on the value of the science and the dark skies that enable it.

The rapidly expanding commercial interest in large constellations of communications and other satellites in low earth orbit poses a different and possibly more significant threat to dark-sky astronomy. The implications are only beginning to be understood. At best, the impact is the loss of geometric area impacted by the bright streaks of reflected sunlight. Detailed analysis and modeling is required for specific use cases to know whether there are more serious impacts from the masking of an unusable area in an image, or the unremovable contribution of reflected sunlight to multi-object spectra. Unlike sources of ground-based light pollution requiring cooperation and control by local regulation, limiting the strongly negative impact of satellite constellations requires cooperation and possible regulation at national and international level. Again, astronomers must make the case that access to the dark night sky is essential for preservation of key observatory locations otherwise not impacted by terrestrial sources. And the astronomy community must look to align with broader communities concerned with long-term sustainability and the protection of the commons of the near-earth environment.

Open Access This article is licensed under a Creative Commons Attribution 4.0 International License, which permits use, sharing, adaptation, distribution and reproduction in any medium or format, as long as you give appropriate credit to the original author(s) and the source, provide a link to the Creative Commons licence, and indicate if changes were made. The images or other third party material in this article are included in the article's Creative Commons licence, unless indicated otherwise in a credit line to the material. If material is not included in the article's Creative Commons licence and your intended use is not permitted by statutory regulation or exceeds the permitted use, you will need to obtain permission directly from the copyright holder. To view a copy of this licence, visit http:// creativecommons.org/licenses/by/4.0/. 


\section{References}

Aubé M, Franchomme-Fossé L, Robert-Staehler P, Houle V (2005) Light pollution modelling and detection in a heterogeneous environment: toward a night-time aerosol optical depth retrieval method. Proc SPIE 5890:589012. https://doi.org/10.1117/12.615405

Aubé M, Fortin N, Turcotte S, García B, Mancilla A, Maya J (2014) Evaluation of the sky brightness at two Argentinian astronomical sites. PASP 126:1068. https://doi.org/10.1086/679227

Aubé M, Simoneau A, Wainscoat R, Nelson L (2018) Modelling the effects of phosphor converted LED lighting to the night sky of the Haleakala Observatory, Hawaii. MNRAS 478:1776. https://doi.org/ 10.1093/mnras/sty1143

Bará S, Aubé M, Barentine J, Zamorano J (2020a) Magnitude to luminance conversions and visual brightness of the night sky. MNRAS 493:2429

Bará S, Falchi F, Furgoni R, Lima RC (2020b) Fast Fourier-transform calculation of artificial night sky brightness maps. JQSRT 240. https://doi.org/10.1016/j.jqsrt.2019.106658

Barentine J et al (2018) Skyglow changes over Tucson, Arizona, resulting from a municipal LED street lighting conversion. JQSRT 212:10. https://doi.org/10.1016/j.jqsrt.2018.02.038

Benn C, Ellison SL (2007) La Palma Night Sky Brightness, La Palma Technical Note 115, http://www. ing.iac.es/Astronomy/observing/conditions/skybr/skybr.html

Cayrel R (1979) Report of Commission 50. Identification and protection of existing and potential observatory sites. Transact IAU 17(1):215-223. https://doi.org/10.1017/S0251107X00010798

Cinzano P, Falchi F (2012) The propagation of light pollution in the atmosphere. MNRAS 427:3337. https://doi.org/10.1111/j.1365-2966.2012.21884.x

Cinzano P, Falchi F, Elvidge CD (2001) The first World Atlas of the artificial night sky brightness. MNRAS 328:689. https://doi.org/10.1046/j.1365-8711.2001.04882.x

Duriscoe DM (2013) Measuring anthropogenic sky glow using a natural sky brightness model. PASP 125:1370. https://doi.org/10.1086/673888

Duriscoe DM (2016) Photometric indicators of visual night sky quality derived from all-sky brightness maps. JQSRT 181:33-45. https://doi.org/10.1016/j.jqsrt.2016.02.022

Duriscoe DM, Luginbuhl CB, Elvidge (2014) The relation of outdoor lighting characteristics to sky glow from distant cities. Light Res Technol 46:35. https://doi.org/10.1177/1477153513506729

Falchi F (2011) Campaign of sky brightness and extinction measurements using a portable CCD camera. MNRAS 412:33. https://doi.org/10.1111/j.1365-2966.2010.17845.x

Falchi F et al (2016) The new world atlas of artificial night sky brightness. Sci Adv 2:e1600377. https:// doi.org/10.1126/sciadv.1600377

Garstang R (1986) Model for artificial night-sky illumination. PASP 98:364. https://doi.org/10.1086/ 131768

Garstang RH (1989a) Night-sky brightness at observatories and sites. PASP 101:306. https://doi.org/10. $1086 / 132436$

Garstang RH (1989b) The status and prospects for ground-based observatory sites. ARAA 27:19. https:// doi.org/10.1146/annurev.aa.27.090189.000315

Garstang RH (1991) Dust and light pollution. PASP 103:1109. https://doi.org/10.1086/132933

Green R (2012) IAU Resolution 2009 B5 - Commission 50 Draft Action Plan - Presentation and Discussion. Proc IAU 10(H16):754-755. https://doi.org/10.1017/S1743921314013362

Hainaut OR, Williams AP (2020) Impact of satellite constellations on astronomical observations with ESO telescopes in the visible and infrared domains. A\&A 636:A121. https://doi.org/10.1051/0004$6361 / 202037501$

Hall S (2019) After SpaceX Starlink Launch, a Fear of Satellites that Outnumber All Visible Stars. New York Times, https://www.nytimes.com/2019/06/01/science/starlink-spacex-astronomers.html

Hampf D, Rowell G, Wild N, Sudholz T, Horns D, Tluczykont M (2011) Measurement of night sky brightness in southern Australia. ASR 48:1017. https://doi.org/10.1016/j.asr.2011.05.010

Hänel A et al (2018) Measuring night sky brightness: methods and challenges. JQSRT 205:278. https:// doi.org/10.1016/j.jqsrt.2017.09.008

IDA (2002) Outdoor lighting code handbook and USA pattern code. International Dark-Sky Association. https://www.darkskysociety.org/handouts/idacodehandbook.pdf

IDA (2010) Visibility, environmental, and astronomical issues associated with blue-rich white outdoor lighting. International Dark-Sky Association. http://darkskycy.com/resources/ida-publications 
Kocifaj M (2007) Light-pollution model for cloudy and cloudless night skies with ground-based light sources. Appl Opt 46:3013. https://doi.org/10.1364/AO.46.003013

Kocifaj M, Bará S (2019) Two-index model for characterizing site-specific night sky brightness patterns. MNRAS 490:1953. https://doi.org/10.1093/mnras/stz2769

Kocifaj M, Solano-Lamphar H, Videen G (2019a) Night-sky radiometry can revolutionize the characterization of light-pollution sources globally. PNAS 116:7712. https://doi.org/10.1073/pnas. 1900153116

Kocifaj M, Wallner S, Solano-Lamphar H (2019b) An asymptotic formula for skyglow modelling over a large territory. MNRAS 485:2214. https://doi.org/10.1093/mnras/stz520

Kocifaj M, Kundracik F, Barentine J, Bará S (2021) The proliferation of space objects is a rapidly increasing source of artificial night sky brightness. MNRAS Lett 504:L40-L44. https://doi.org/10. 1093/mnrasl/slab030

Kolláth K, Kolláth Z (2020) On the feasibility of using ceilometer backscatter profile as input data for skyglow simulation. JQSRT 253:107158. https://doi.org/10.1016/j.jqsrt.2020.107158

Kolláth Z, Cool A, Jechow A, Kolláth K, Száz D, Tong K-P (2020) Introducing the dark sky unit for multi-spectral measurement of the night sky quality with commercial digital cameras. JQSRT 253:107162. https://doi.org/10.1016/j.jqsrt.2020.107162

Krisciunas K (1997) Optical night-sky brightness at Mauna Kea over the course of a complete sunspot cycle. PASP 109:1181. https://doi.org/10.1086/133993

Krisciunas K, Semler DR, Richards J, Schwarz HE, Suntzeff NB, Vera S, Sanhueza P (2007) Optical sky brightness at Cerro Tololo Inter-American Observatory from 1992 to 2006. PASP 119:687. https:// doi.org/10.1086/519564

Krisciunas K, Bogglio H, Sanhueza P, Smith MG (2010) Light pollution at high zenith angles, as measured at Cerro Tololo Inter-American Observatory. PASP 122:373. https://doi.org/10.1086/ 651246

Kyba C, Kuester T, Sánchez de Miguel A, Baugh K, Jechow A, Hölker F, Bennie J, Elvidge C, Gaston K, Guanter L (2017) Artificially lit surface of Earth at night increasing in radiance and extent. Sci Adv 3(11):e1701528. https://doi.org/10.1126/sciadv.1701528

Lockwood W (2002) The Lowell Observer

Luginbuhl CB, Walker CE, Wainscoat RJ (2009a) Lighting and astronomy. Phys Today 62(12):32. https://doi.org/10.1063/1.3273014

Luginbuhl CB, Lockwood GW, Davis DR, Pick K, Selders J (2009b) From the ground up I: Light pollution sources in Flagstaff, Arizona. PASP 121:185. https://doi.org/10.1086/597625

Luginbuhl CB, Duriscoe DM, Moore CW, Richman A, Lockwood GW, Davis DR (2009c) From the ground up II: Sky glow and near-ground artificial light propagation in Flagstaff, Arizona. PASP 121:204. https://doi.org/10.1086/597626

Luginbuhl CB, Boley PA, Davis DR, Duriscoe DM (2012) The effects of lamp spectral distribution on sky glow over observatories. Proc IAU 10(H16):756. https://doi.org/10.1017/S1743921314013374

Luginbuhl CB, Boley PA, Davis DR (2014) The impact of light source spectral power distribution on sky glow. JQSRT 129:21. https://doi.org/10.1016/j.jqsrt.2013.12.004

Massey P, Foltz CB (2000) The spectrum of the night sky over Mount Hopkins and Kitt Peak: changes after a decade. PASP 112:566. https://doi.org/10.1086/316552

McDowell JC (2020) The low earth orbit satellite population and impacts of the SpaceX Starlink constellation. Astrophys J 892:L36. https://doi.org/10.3847/2041-8213/ab8016

MMA (2021) Anteproyecto de norma de emisión elaborado a partir de la revisión del Decreto Supremo ${ }^{\circ}$ 43, de 2012, del Ministerio del Medioambiente, que establece norma de emisión para la regulación de la contaminación lumínica. https://consultasciudadanas.mma.gob.cl/portal/consulta/104

Müller A, Wuchterl G, Sarazin M (2011) Measuring the night sky brightness with the lightmeter. Rev Mex AA (Ser Conf) 41:48

NPS (2006) Management Policies 2006. U.S. Department of the Interior / National Park Service. https:// www.nps.gov/subjects/policy/upload/MP_2006.pdf

Neugent KF, Massey P (2010) The spectrum of the night sky over Kitt Peak: changes over two decades. PASP 122:1246. https://doi.org/10.1086/656425

Patat F (2008) The dancing sky: 6 years of night-sky observations at Cerro Paranal. A\&A 481:575. https://doi.org/10.1051/0004-6361:20079279

Pedani M (2009) Sky surface brightness at Mount Graham: UBVRI science observations with the Large Binocular Telescope. PASP 121:778. https://doi.org/10.1086/603605 
Pipkin A, Duriscoe D, Luginbuhl C (2017) Measuring the color and brightness of artificial sky glow from cities using an all-sky imaging system calibrated with astronomical methods in the Johnson-Cousins B and V photometric systems, American Astronomical Society, AAS Meeting \#229, id.236.20.

Ragazzoni R (2020) The surface brightness of megaconstellation satellite trails on large telescopes. PASP 132:114502. https://doi.org/10.1088/1538-3873/abaca8

Riddle RL et al (2008) An analysis of light pollution at the Thirty Meter Telescope candidate sites. Proc SPIE 7012:701223. https://doi.org/10.1117/12.787295

Roach FE, Gordon JL (1973) The light of the night sky. Reidel, Dordrecht. https://doi.org/10.1007/97894-010-2553-9

Sánchez SF, Aceituno J, Thiele U, Pérez-Ramírez D, Alves J (2007) The night sky at Calar Alto Observatory. PASP 119:1186. https://doi.org/10.1086/522378

Taylor VA, Jansen RA, Windhorst RA (2004) Observing conditions at Mount Graham: Vatican Advanced Technology Telescope UBVR sky surface brightness and seeing measurements from 1999 through 2003. PASP 116:762. https://doi.org/10.1086/422929

Tregloan-Reed J, Otarola A, Ortiz E, Molina V, González AR, Colque JP, Unda-Sanzana E (2020) First observations and magnitude measurement of Starlink's Darksat. A\&A 637:L1. https://doi.org/10. 1051/0004-6361/202037958

Tyson JA, Ivezić Ž, Bradshaw A, Rawls ML, Xin B, Yoachim P, Parejko J, Greene J, Sholl M, Abbott TMC, Polin D (2020) Mitigation of LEO satellite brightness and trail effects on the Rubin Observatory LSST. AJ 160:226. https://doi.org/10.3847/1538-3881/abba3e

Walker MF (1973) Light pollution in California and Arizona. PASP 85:508. https://doi.org/10.1086/ 129496

Walker A (1987) Sky brightness at CTIO. NOAO Newsletter No. 10. https://hdl.handle.net/2027/mdp. 39015019262131

Walker A, Schwarz H (2007) Night Sky Brightness at Cerro Pachon. http://www.ctio.noao.edu/site/ pachon_sky/

Walker C, Hall J, Allen L, Green R, Seitzer P, Tyson T et al (2020a) Impact of satellite constellations on optical astronomy and recommendations toward mitigations. Bull AAS 52(2). https://doi.org/10. $3847 / 25$ c2cfeb.346793b8

Walker C et al (2020b) Workshop on Dark and Quiet Skies for Science and Society. Report and recommendations. IAU. https://www.iau.org/static/publications/dqskies-book-29-12-20.pdf

Wall M (2019) SpaceX's 60-Satellite Launch Is Just the Beginning for Starlink Megaconstellation Project, Space.com, https://www.space.com/spacex-starlink-satellites-launch-just-beginning.html.

Yao S et al (2012) The site conditions of the Guo Shou Jing Telescope. RAA 12:772. https://doi.org/10. 1088/1674-4527/12/7/005

Zhang J-C, Ge L, Lu X-M, Cao Z-H, Chen X, Mao Y-N, Jiang X-J (2015) Astronomical observing conditions at Xinglong Observatory from 2007 to 2014. PASP 127:1292. https://doi.org/10.1086/ 684369

Zou H, Zhou X (2011) The i-band sky brightness and transparency at Dome A, Antarctica. arXiv e-prints arXiv:1101.2362

Publisher's Note Springer Nature remains neutral with regard to jurisdictional claims in published maps and institutional affiliations.

\title{
Authors and Affiliations
}

\section{Richard F. Green ${ }^{1}$ (D) - Christian B. Luginbuhl ${ }^{2} \cdot$ Richard J. Wainscoat $^{3}$. Dan Duriscoe ${ }^{4}$}

\author{
Richard F. Green \\ rgreen@arizona.edu \\ Christian B. Luginbuhl \\ starlightcbl@msn.com
}


Richard J. Wainscoat

rjw@hawaii.edu

Dan Duriscoe

dan@nightskymetrics.com

1 Steward Observatory, University of Arizona, 933 N. Cherry, Tucson, AZ 85721-0065, USA

2 Flagstaff Dark Skies Coalition, P.O. Box 1892, Flagstaff, AZ 86002, USA

3 Institute of Astronomy, University of Hawaii, 2680 Woodlawn Dr., Honolulu, HI 96822, USA

4 Night Sky Metrics LLC, 21781 Ventura Blvd \#1009, Woodland Hills, CA 91364, USA 